\title{
HIGH-RESOLUTION, NON-CONTACT ANGULAR MEASUREMENT SYSTEM FOR PSA/RSA
}

\author{
A Thesis \\ presented to \\ the Faculty of California Polytechnic State University, \\ San Luis Obispo
}

\author{
In Partial Fulfillment \\ of the Requirements for the Degree \\ Master of Science in Industrial Engineering
}

by

Ronald Sloat

March 2011 
(C) 2011

Ronald Sloat

ALL RIGHTS RESERVED 


\section{COMMITTEE MEMBERSHIP}

TITLE: High-Resolution, Non-Contact Angular Measurement System for PSA/RSA AUTHOR: Ronald Sloat

SUBMITTED: March, 2011

COMMITTEE CHAIR: $\quad$ Dr. Jianbiao Pan, Associate Professor, IME

COMMITTEE MEMBER: Dr. Jose Macedo, Department Chair/Professor, IME

COMMITTEE MEMBER: Dr. John Ridgely, Associate Professor, ME 


\section{ABSTRACT \\ High-Resolution, Non-Contact Angular \\ Measurement System for PSA/RSA \\ Ronald Sloat}

A non-contact angular measurement system for Pitch Static Attitude (PSA) and Roll Static Attitude (RSA) of hard disk drive sliders is designed and built. Real-time sampling at over $15 \mathrm{KHz}$ is achieved with accuracy of $+/-0.05$ degrees over a range of approximately 2-3 degrees. Measuring the PSA and RSA is critical for hard drive manufacturers to control and improve the quality and reliability of hard drives. Although the hard drive industry is able to measure the PSA and RSA at the subassembly level at this time, there is no system available that is able to measure PSA/RSA at the final assembly level. This project has successfully demonstrated a methodology that the PSA/RSA can be reliably measured in-situ using a laser and position sensitive detector (PSD) technology. A prototype of the measurement system has been built using simple and inexpensive equipment. This device will allow a continuous measurement between the parked position on the ramp and the loading position just off of the disk surface. The measured data can be used to verify manufacturing processes and reliability data.

Keywords: Disk, Drive, PSA, RSA, pitch, roll, static, attitude, dynamic, slider, measurement, laser, PSD, position, sensitive, detector, diode 


\section{ACKNOWLEDGEMENTS}

I would like to thank Western Digital Corporation for providing the funding and motivation for this project. Special thanks to David Renuart, Minh-Nhat Le, Matthew Brown, Keith Berding and David Bagaoisan for their time, experience, and knowledge. Thanks to Dr. John Ridgely, whose classes made this project manageable, and to Dr. Jose Macedo for participating in the committee for my defense. Thanks to the Cal Poly IME Department for their expertise and help in fabricating parts for this project, and to my project partner Doug Smith for his time and help. And many thanks to my thesis advisor, Dr. Jianbiao Pan, whose guidance and help throughout college led me here, and whose experience and knowledge have been invaluable. 


\section{TABLE OF CONTENTS}

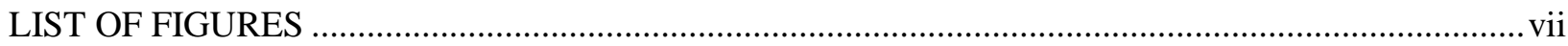

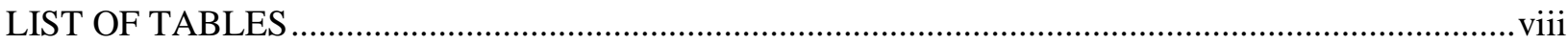

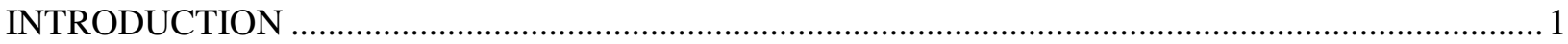

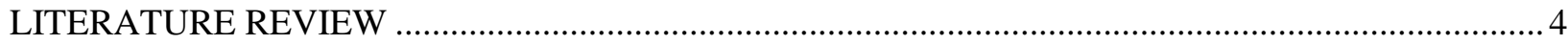

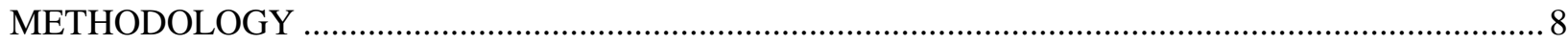

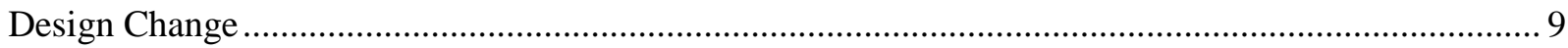

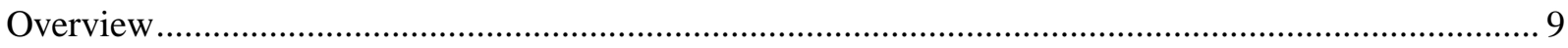

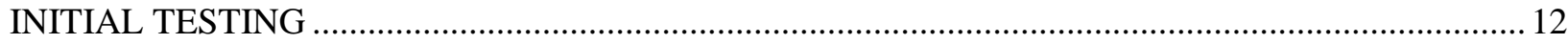

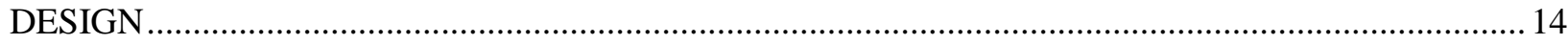

Hardware

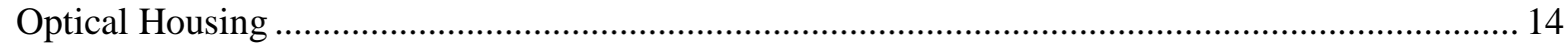

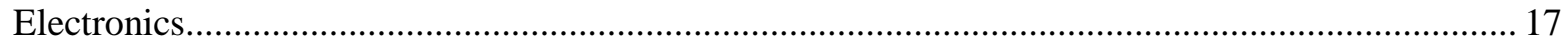

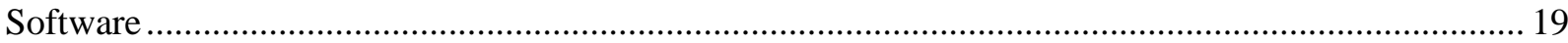

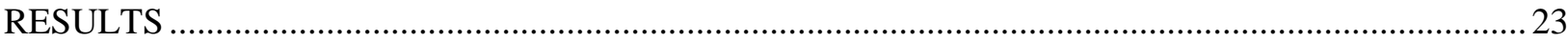

CONCLUSION

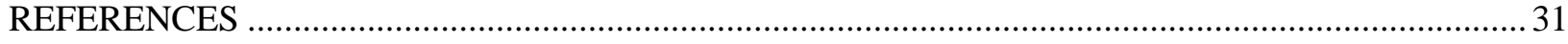

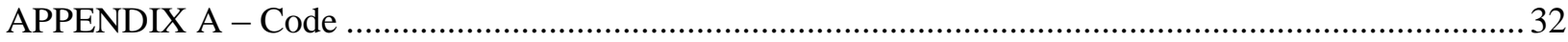

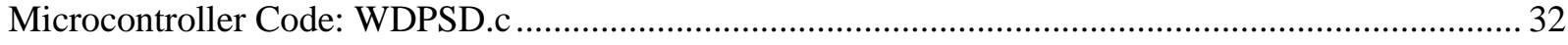

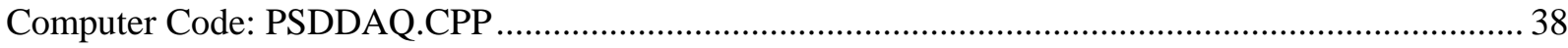

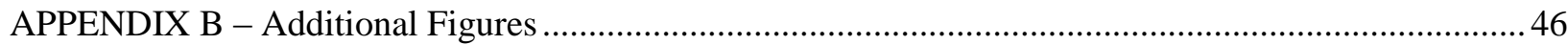




\section{LIST OF FIGURES}

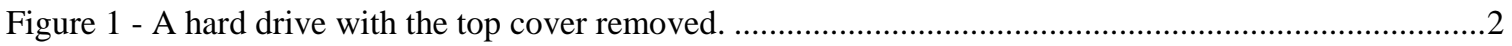

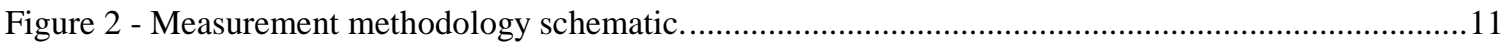

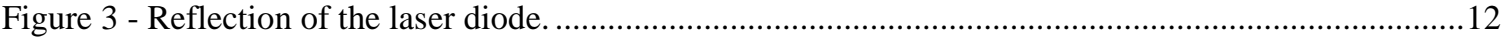

Figure 4 - PSD measurement method operating schematic...................................................................13

Figure 5 - Exploded view of the optical housing................................................................................14

Figure 6 - Geometrical measurement error in reflected laser. ...................................................................15

Figure 7 - Spherical aberration occurring in a spherical lens. ................................................................16

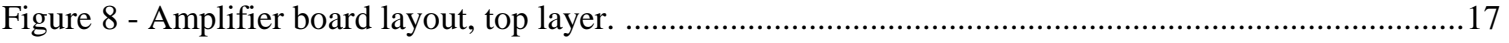

Figure 9 - Assembled circuit boards...................................................................................................18

Figure 10 - Microcontroller board layout, top layer. ………...............................................................19

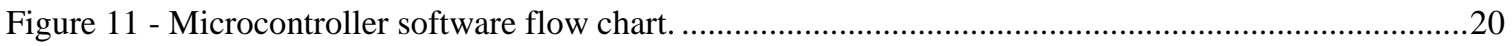

Figure 12 - Data acquisition software screenshot from the PC. ................................................................

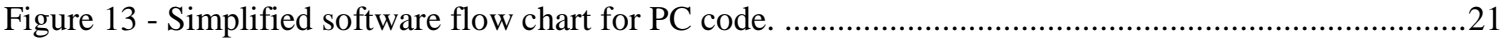

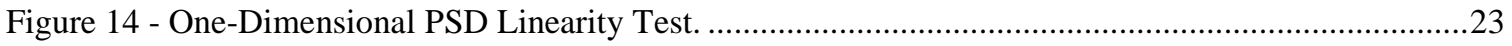

Figure 15 - Two-Dimensional PSD output showing spherical abberation of the focusing lens. ...................24

Figure 16 - Validation of the measurement method using the disk surface on the 2D PSD........................24

Figure 17 - Validation of the measurement method on the slider surface on the 2D PSD...........................25

Figure 18 - Measurement system sensitivity as a function of proximity to the measurement surface. ….....26

Figure 19 - Measurement system output from the disk surface, with averaging. ..........................................2

Figure 20 - Measurement system output from the slider surface, with averaging.........................................27

Figure 21 - Still disk surface measurement, showing a constant reading with few errors. ...........................28

Figure B 1 - Exploded rear view of the optical housing.

Figure B 2 - Electronics schematic of microcontroller and amplifier. ............................................................48 


\section{LIST OF TABLES}

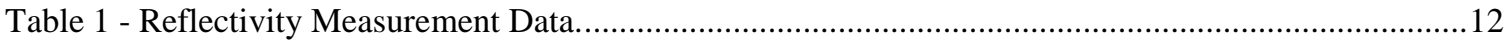

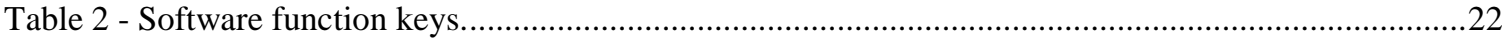




\section{INTRODUCTION}

Hard drives are a very complicated piece of equipment present in almost all computers. They provide a solution to store data that is non-volatile and quite compact. As the demand for performance increases in computers, so too is the demand for storage to hold the increasing number and size of programs and media. The demand for storage has increased exponentially with the capabilities of computers being able to handle 3D graphics, complicated Finite Element Analysis and video editing. With the physical size of the disks fixed by industry standards, the only way of getting more data to fit is by putting more data in the same space. This requires extremely small tracks of data to be recorded and read off of the disk.

The mechanism used to read and write on the disk media is called the head. The head is attached to a small block called the slider. The slider's purpose is to provide a bearing that will float the slider above the surface of the disk. If the slider were to ride on the disk, tiny scratches would develop that could degrade the magnetic performance of the disk. The further the head flies from the platter, the larger the magnetic field must be for reliable storage, and the less data can be stored per square inch. The closer the head flies from the platter, the smaller the magnetic field can be, and the more data can be stored.

For the proper function of a hard drive, the read and write heads must fly at a very small distance above the magnetic platters. This distance can be as small as several nanometers. To ensure a smooth fly height, the slider creates an air bearing to buffer itself from the platters. This air bearing is critical to the performance of the drive and to prevent the slider from scratching the platter surface. To create this bearing, the head must have a certain attitude, or angle. The two angles of concern are called the pitch and roll [1]. By providing a small attitude in the proper direction, the head can catch air as it is flown over the platters to support itself. Too much angle can cause the trailing edge of the head to drag on the surface, causing scratches and ruining the 
disk [2], or cause it to fly too far from the surface, thereby decreasing the effectiveness of the magnetic field used to record on the medium.

The measurement of the angles of the heads is a non-trivial task. The air-bearing surface is a mere $1.1 \times 0.8 \mathrm{~mm}$. At the pre-assembly level, various optical and laser techniques can be used to measure the angles because they can be mounted and moved to any position to facilitate the measurement. Once the drive is assembled, there is little area to make the measurement since it is enclosed in an aluminum chassis. Using expensive glass disks, the pitch and roll can be measured over the disk surface using laser interferometry. Even then, the drive is not fully assembled, nor is it assembled with the correct components. It is currently unknown how drastically the angle changes after assembly, and this increases the uncertainty of the failure analysis. In a typical drive, there can be about eight heads, two per disk platter. The measurement system would ideally be able to measure each head despite the different orientations. Figure 1 shows a typical hard drive.

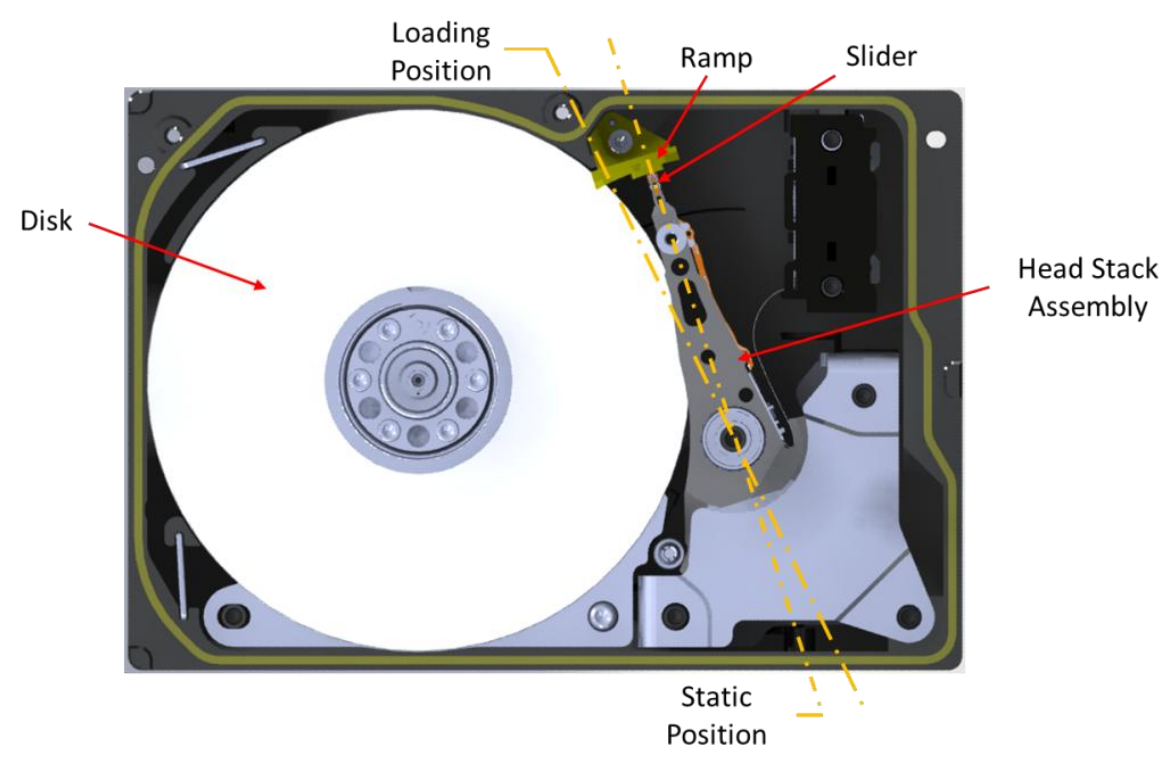

Figure 1 - A hard drive with the top cover removed.

The motivation for this project revolves around the need for failure analysis of the hard drives. Almost every parameter is recorded to get a reliability and performance estimate of the drives. 
Consumers demand high reliability to keep data safe. However, once assembled, little information is received about the internals of the drive and only after the drive fails or is disassembled can information be obtained. This project would partly bridge the gap and allow an additional variable to be measured to see how it affects hard drive performance and reliability. Information on the optimal orientation has already been gathered, but that information cannot be fully utilized in manufacturing due to the variation and the inability to measure the parameters.

The objective of this project is to measure the angles of the slider at two positions. The first position is called the static position. This is when the sliders are parked on the ramp, off of the disk surface. The second position is when the sliders are in the loading position. This occurs when the sliders are moving towards the disk and are about to develop the air bearing. This project will help to characterize the drive better with more knowledge to how the sliders behave off of the disk.

The project can be broken down into three main stages. The first stage is researching the various measurement methods to determine a method that has a resolution of $+/-0.05$ degrees that can be performed in situ. Once the measurement methods have been identified, they will be evaluated to determine which one is the most feasible method for in-situ measurement. The proposed measurement system will then be experimentally verified.

The second stage will be designing the fixturing, tooling, electronics and methods to perform the test. The goal would be to mount a finished drive onto the apparatus and perform a nondestructive test of the drive. Slight modification of the drive may be necessary to access the sliders, but this test would not be performed on every drive. Automation and measurement speed are not an issue at this point, and a manual testing design will be adequate.

The third stage will be to build, assemble and test the apparatus. Due to the sensitivity of this measurement, the fixturing will need to be rigid, and the entire assembly will probably need to be mounted onto a granite slab to reduce environmental vibrations interfering with the testing. 


\section{LITERATURE REVIEW}

Measurement of tilt and inclination of a plane to high-resolution is not a simple task. Big, solid surfaces may allow a large, bulky instrument to sit on top for the measurement, but small, flexible parts cannot have large contact forces. Therefore it is a requirement to have a non-contact way of measuring the inclination. A variety of methods exist that include optical, laser and magnetic sensors. All have their pros and cons and not all are suited to the small area of the hard drive slider.

One of the most common methods of measuring small distances without contact is by interferometry [3]. Two fundamental methods exist: heterodyne detection and homodyne detection. Homodyne detection uses a beam of light that is split and reflected off of two different parts. One is reflected off of a reference mirror and hits a photo detector. The other beam of light is reflected off of the part of interest. These two beams, once combined at the photo detector, allow a determination of a fringe order. This fringe order determines the fractional amount of the wavelength of the light that differs from the reference beam. This directly relates to the position of the part. If the device is set up and calibrated properly, any change in the phase of the laser as it hits the photo detector can be detected, producing resolutions on the nanometer scale.

Heterodyne detection used in interferometry produces a similar effect as homodyne detection with the added benefit of better signal-to-noise ratio. This method employs the same setup as homodyne detection, but one beam is modulated before being detected. Typically, the frequency is modulated. This produces a beat frequency relative to the difference between the frequencies of the beams. Since the modulated frequency is a known value, any change in the beat frequency is accurately known. Most commercial interferometers utilize the heterodyne detection scheme.

Accurate methods of measuring distance can be used to measure inclination of a flat surface. With two distance measurements along a surface, the surface slope can be calculated. This does not provide information about the whole surface, but rather just in the direction of the vector 
created by the two points. Unless the surface can be characterized before measurement, three points are needed to define the plane of the surface [4]. This can allow accurate determination of the slope in any direction along the plane. Utilizing the mathematics of a plane, it is simple to determine three points and calculate an angle in any direction.

A similar device used for measuring displacement is a laser displacement sensor [5]. Rather than needing an optical reference and calculating the interference between incident light waves, a laser displacement sensor shines a laser onto a surface and a calibrated focusing system focuses onto the laser spot. From the focal distance measured by the system, the actual displacement from the sensor can be measured to sub-micron accuracies. This system is much simpler than an interferometry setup in the sense that the laser light does not need to be combined to detect phase. This means that the laser can be incident to the surface as angles smaller than 90 degrees. Though not as accurate as an interferometry system, the flexibility is superior.

Measuring displacements to obtain angular information requires taking two or more measurements with the measurement sensor as stable and fixed as possible so that any difference in measurements correlates to the orientation of the object. With an interferometer or laser displacement sensor, two or three points are needed to calculate a line or plane. The orientation of the line or plane can be used to determine the slope in the directions desired. Another device, called a confocal laser scanning displacement meter [6] can scan and measure displacement over a small range without moving the sensor. By using an oscillator and a tuning fork, small vibrations change the focal path of the instrument, allowing measurements of displacement along a line of several millimeters. Angularity can be directly measured from these data points, and by changing the orientation of the device and measuring again, enough data points can be obtained to define the entire surface.

Lasers can be used in a variety of metrological equipment. One such method is known as laser reflectometry. This method employs a laser that is shone onto a surface, and the reflection of the 
laser is measured. This reflection typically equates to an orientation of the object, but can also be setup to measure distances. Measuring the displacement caused by the reflection of the laser can be done is several ways.

A CCD sensor [7] is essentially an array of tiny photo detectors mounted onto a small package or IC. Sensitivities vary, but when photons of light fall onto a photo-detector, the individual detector can give a signal proportional to the light intensity and the position of the individual detector can be calculated. This gives two simultaneous readings, but is limited due to the discrete nature of the photo detectors. Light impinging between detectors cannot be measured. With a good design, CCD sensors can give very good data with very good accuracy.

Another sensor that can be used to measure displacement of a laser beam is a position sensitive detector, or PSD [8]. This is essentially a large photodiode with a constant resistivity over its surface, built with a P-doped region resting on top of an intrinsic semiconductor region, resting on an $\mathrm{N}$-doped region. A spot of light incident on the diode surface causes current to flow to the electrodes inversely proportional to the location of the light on the surface. Due to the analog nature of the device, any change in displacement can be picked up because there are no discrete cells. However, due to the small currents generated and noise in the system, typical resolutions are around 1 micron. These sensors come in one-dimensional and two-dimensional arrays, allowing for flexibility is measurements. An important part of a PSD sensor is incorporating the electronics required to amplify the signals, and high-quality, low-noise instrumentation amplifiers are normally used to ensure good accuracy.

As digital optical technology advances, high-resolution photographs can give accurate data about an object. This can be used as a non-contact way of measuring the extremely small surface angles of the hard disk sliders. Typical cameras can achieve accuracies close to $+/-0.1^{\circ}$ [9]. Many test setups have used optical cameras in addition to other measurement methods. Due to the confined space, cameras are not well-suited for the measurement. 
A method in use in the hard drive industry to measure the orientation of the sliders is called shadow-cast optoelectronic gauging [10]. This principle uses collimated light to cast a shadow of the part on a photo-detector array. Typically this light is infrared. Utilizing highly sensitive control systems for the positioning of the sensors, rotating the sensing array creates a distribution of the shadow profile on the sensor. The peak point on the shadow distribution relates to the actual position or orientation of the part under test.

Hard drive technology has changed in recent years, but the write head still utilizes induction to create the magnetic field. Magnetoresistive materials have been discovered that change their resistance based on the strength and direction of the magnetic field that passes through it [11]. Originally discovered in 1856 by Lord Kelvin, these materials have been incorporated into integrated circuits that are commercially available today and can measure a full $360^{\circ}$ rotation of a magnet positioned above the sensor. These devices can achieve accuracies limited only by the linearity of the device and the precision of the other electronic devices used. Many use 13-bit analog-to-digital convertors and have a resolution of $0.022^{\circ}$ accordingly. This is a non-contact measurement method that relies on a magnet with a north- and south-pole parallel to the sensor to produce the change in direction of the magnetic field. In non-rotating applications, the change in resistance can be related to the change in linear displacement, but are limited in range.

Two electrically conductive surfaces separated by a non-conductive dielectric material exhibit a phenomenon known as capacitance. It is well known that the capacitance is inversely proportional to the distance between the conductive surfaces. By utilizing this principle, measurements of displacement can be made [12]. Unlike laser measurement systems which can be focused to a small point, capacitive sensors measure over an area the size of the sensing head, averaging throughout. Multiple measurements on a small surface are not possible with a capacitive sensor. Measurement accuracies on the nanometer scale can be expected with high-quality capacitive sensors. 


\section{METHODOLOGY}

Determining the optimal measurement solution is difficult. Based on the current methods available, many fail to be able to measure such a small surface or to be inserted into such a small space. Of the remaining solutions, four criteria will need to be met as provided by Western Digital. These are:

- Minimum resolution of $+/-0.05^{\circ}$

- Repeatable

- Accurate to $+/-0.05^{\circ}$

- Perform in-situ

Additional requirements such as measurement simplicity, ease of manufacturing and cost also play a significant role.

The measurement system will take three separate readings, one while the head stack assembly is parked on the ramp, one when the sliders are in the loading position and one measurement of the disk surface itself to provide a reference surface for the two measurements. This will be repeated for each slider in the drive.

Of the many methods examined, most require a sensing unit to be mounted fairly close to the sliders. This presents a problem in the constrained area of the drive. For this reason, we focused on the two laser-based measurement methods: the PSD and the laser displacement sensor.

The PSD can determine the exact location of a laser spot on its surface by producing currents inversely proportional to the distance from the centroid of the laser spot to the electrodes on its surface. This current can be converted into a voltage and measured. The ratio of these currents (now voltages) determines the spatial location of the laser on the PSD sensor. Utilizing a twodimensional sensor, both the pitch and roll can be determined from the one measurement. This provides a very robust design, without the need for multiple measurements to obtain the data.

The laser displacement sensor uses a laser and a camera to determine the distance between the measurement unit and a surface. By focusing the camera lens on the spot, a very accurate distance 
is produced. To adapt this to our project, three data points would be needed. By obtaining three points on the surface of the slider, a plane can be created, giving the necessary pitch and roll angles. One advantage of this system is that a company, Keyence, provides off-the-shelf solutions of this type.

\section{Design Change}

On December $11^{\text {th }}$, 2009, a design review was held at Western Digital in San Jose, California. The PSD method was chosen by the design team, and work began on the design and on validating the measurement method. Furthermore, the scope and workings of the project were revised.

The measurement method would no longer provide two measurements for the purpose of comparison between the static and the dynamic condition. The new method would be a continuous measurement from the static to dynamic condition. This would provide more insight into the orientation of the slider as it exits the ramp and loads onto the disk.

The measurement method would no longer be required to measure every slider in the drive. Primarily the top-most slider in the head stack will be measured, with the possibility of also measuring the bottom-most slider.

With this new change, the laser displacement sensor would require the use of three units, each taking measurements in tandem to produce a continuous measurement. Due to the cost of the displacement sensors, the PSD method was chosen for further investigation.

\section{Overview}

The basis of the project is to measure the orientation of a surface relative to another by means of a reflected beam of laser light. The laser beam on the reference surface will cause a certain displacement on the position sensor. This position will correspond to a virtual flat surface, with no angle of rotation. By taking a second measurement on the surface of interest, any deviation 
from the first measurement's displacement will predict the orientation of the second surface with respect to the first. Figure 2 shows the simplified measurement setup.

This simplifies the demand on the accuracy of the device by performing a relative measurement. An absolute measurement would require an accuracy of design and calibration that would be impractical for this project.

The slider pitch and roll is typically measured with respect to the spinning disks. It is therefore natural to use the spinning disks as the reference surface for this project. All subsequent measurements will be relative to the disks.

The Position Sensitive Detector (PSD) will be used to measure the displacement of the laser beam. It produces variable currents on its output pins that can be used to determine the location of the centroid of the laser spot. This provides easy integration for automated readings. The equations to determine the laser spot position are given below.

$$
\begin{aligned}
& x=\frac{\left(i_{X 2}+i_{Y 2}\right)-\left(i_{X 1}+i_{Y 1}\right)}{i_{X 1}+i_{X 2}+i_{Y 1}+i_{Y 2}} \times\left(\frac{L_{x}}{2}\right) \\
& y=\frac{\left(i_{X 2}+i_{Y 1}\right)-\left(i_{X 1}+i_{Y 2}\right)}{i_{X 1}+i_{X 2}+i_{Y 1}+i_{Y 2}} \times\left(\frac{L_{y}}{2}\right)
\end{aligned}
$$

The variables $x$ and $y$ are the coordinates of the location of the centroid of the laser spot on the surface of the PSD. The currents, denoted $i_{X n}$ and $i_{Y n}$, are the currents flowing through each electrode. The sensing area size, denoted as $L_{x}$ and $L_{y}$, converts the ratio of the currents into the location, usually in microns.

To be able to collect real-time data, a microcontroller will be used to sample, calculate, and transfer data to a computer. A microcontroller proved very useful in the initial round of testing, and it was deemed a better method than buying a dedicated analog to digital converter card from a manufacturer such as National Instruments. 


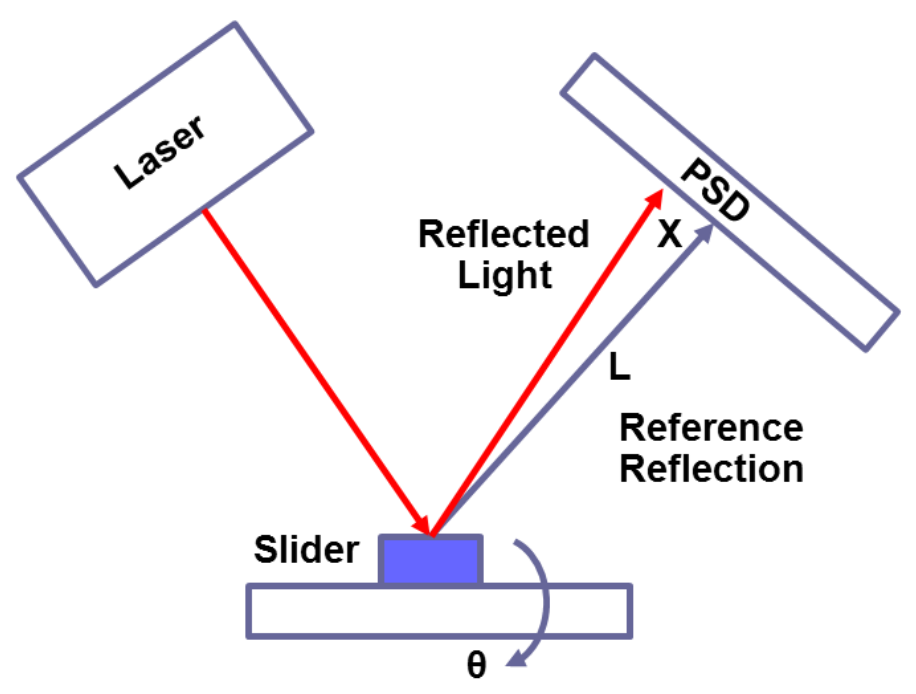

Figure 2 - Measurement methodology schematic.

The most important step is to determine the resolution of the measurement apparatus. The primary purpose is to measure very small angles accurately. This will be done using actual hard drive sliders mounted onto a fixture. The fixture will support the sliders and allow the measuring device to be mounted in any position or orientation needed for the measurement. For all measurements, we will need a baseline reference. This will be accomplished by utilizing the disk surface in the hard drive. The disk surface is already extremely flat, with a roughness of only $1 \AA$, and highly reflective. It is a perfect reference for the orientation of the slider. The angle measurement taken on the disk surface will determine the zero-degree reference that will define the relative attitude of the slider.

The final requirement will be to determine the ability of the method to perform in-situ. Due to space limitations, a setup that requires no modification to the original parts is highly unlikely. Therefore, the least amount of modification will be desired. Certain methods could require large holes cut into the top plate of the drive to allow access while other methods may only need a small hole to allow a laser to be shot into the drive. The feasibility of each method will need to be evaluated quantitatively based on the number of requirements and modifications and also for possible automation in the future. 


\section{INITIAL TESTING}

Preliminary tests were conducted during the project to ensure that the measurement method would perform adequately for the actual measurement. Testing milestones included:

- Surface Reflectivity

- Lens Positioning

- Laser Positioning

Using a Thor Labs optical power meter, the reflectivity of several parts of the head stack assembly were measured. The results are presented in Table 1 below. A typical reflection is shown in Figure 3.

Table 1 - Reflectivity Measurement Data.

\begin{tabular}{lcc}
\hline Surface & Reflected Power & Reflectivity \\
\hline Slider & $90 \mu \mathrm{W}$ & $9 \%$ \\
Slider (Side) & $80 \mu \mathrm{W}$ & $8 \%$ \\
Gimbal & $320 \mu \mathrm{W}$ & $32 \%$ \\
\hline & Laser Power & $1 \mathrm{~mW}$
\end{tabular}

With this data, it is clear that the initial laser power must be fairly large to produce a reasonable amount of power at the PSD. With a 50/50 beam splitter, half of the optical power is lost each time the laser passes through it. A maximum of $25 \%$ of the optical power will hit the PSD. Using the slider surface, only $2-3 \%$ of the optical power can be used for the measurement.

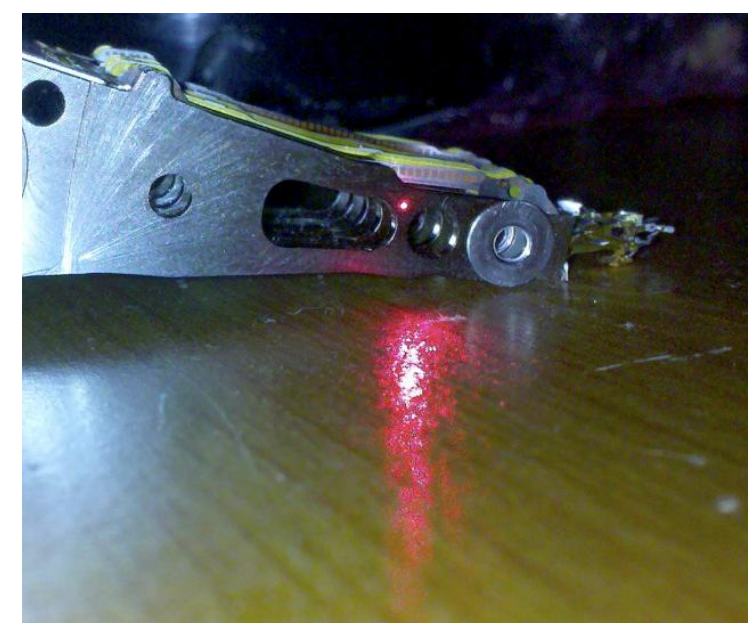

Figure 3 - Reflection of the laser diode. 
The PSD, using $635 \mathrm{~nm}$ red light, has a quantum efficiency of roughly 0.4 Amperes per Watt of incident light. With a saturation current of $0.2 \mathrm{~mA}$, this yields a maximum optical power of $500 \mu \mathrm{W}$. Designing for the disk surface, a near-perfect mirror, a $2 \mathrm{~mW}$ laser diode will be required for maximum current, due to at least $75 \%$ loss through the beam splitter.

To reduce complicated fixture design and setup time for the finished measurement unit, it would be ideal for the laser beam to take the same path for incidence and reflection. To achieve this, a beam splitter is placed between the laser and the measurement surface. When the laser returns to the beam splitter, it diverges at $90^{\circ}$. The PSD is then placed on axis with the new diverged beam path. Figure 4 below shows the operating schematic.

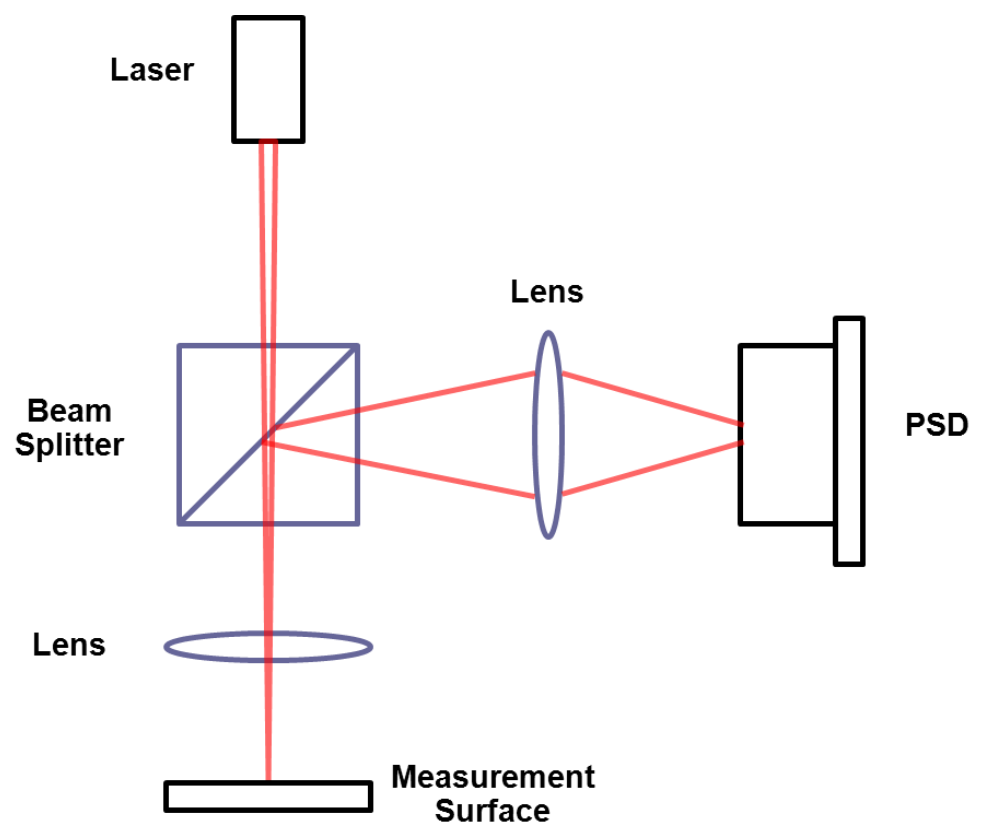

Figure 4 - PSD measurement method operating schematic.

For the optics, it was originally thought that the roughly collimated laser beam would remain collimated until it reached the PSD. During experimental testing, it was seen that the reflected laser was dispersed and diverged at a much greater angle. Additional lenses would be required to recapture and refocus the light onto the PSD surface. Two lenses were added to the design for this purpose. Using two lenses helps avoid using a single, short focal length lens that would introduce greater spherical aberration. 


\section{DESIGN}

\section{Hardware}

\section{Optical Housing}

The optical housing was designed to mount and align every piece of the measurement system to provide an easy to mount unit for the final measurement fixture. The measurement system consists of several components:

- Laser Diode assembly

- Beam splitter

- Refocusing lenses

- PSD

- Amplifier electronics

The housing was designed to be as small and robust as possible. A big emphasis was placed on the ease of machinability. The final design is seen below in Figure 5.

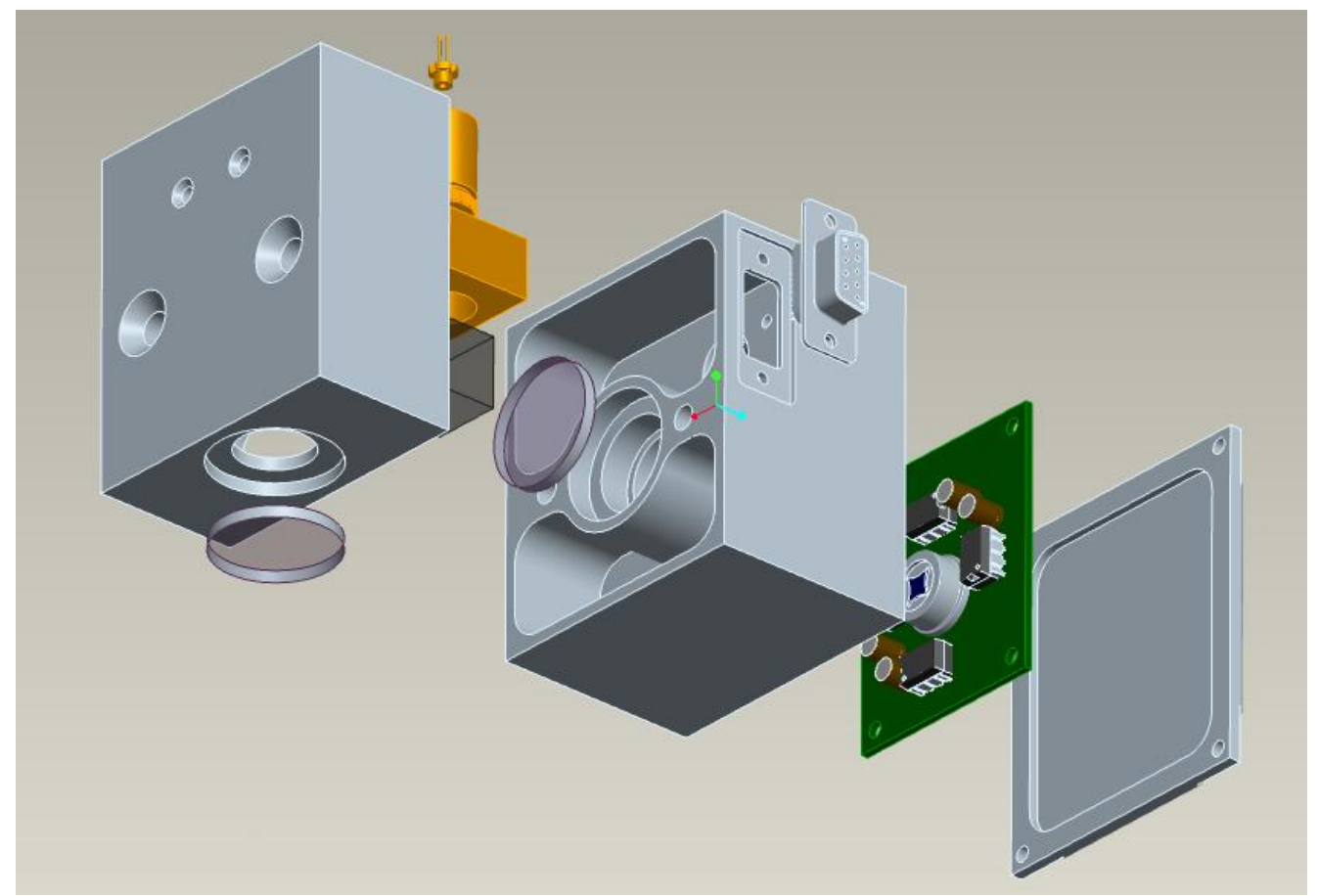

Figure 5 - Exploded view of the optical housing.

Besides keeping the components aligned, the housing controls the beam path length between the measurement surface and the PSD. This length controls the overall measurement range and resolution. In theory, a longer path length produces greater resolution. For a fixed PSD sensor 
size, this results in less measurement range. The lenses on the optical path help focus the laser, but also reduce the effective path length, reducing the measurement resolution.

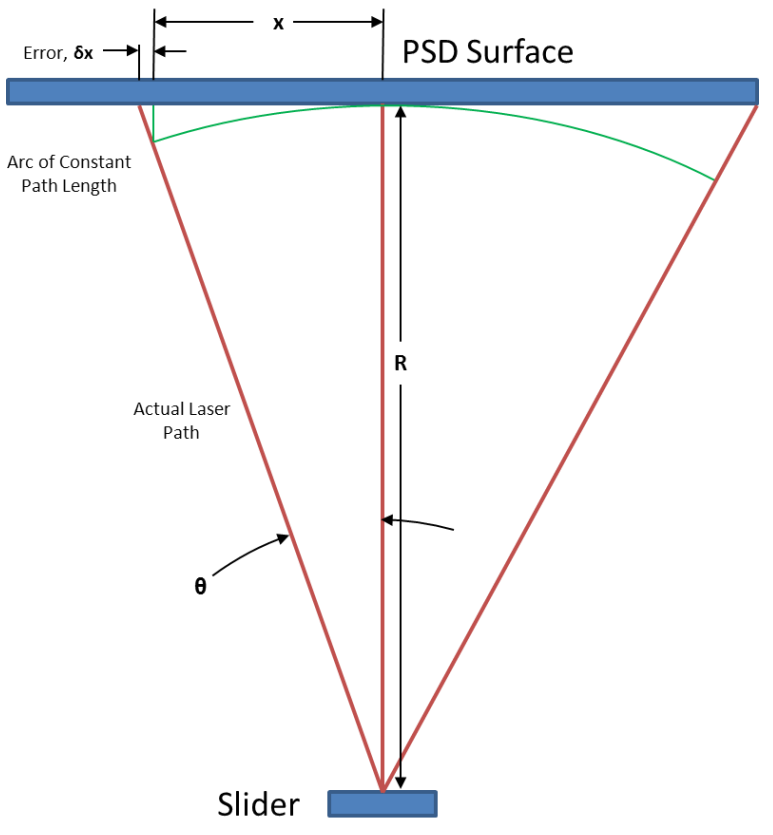

Figure 6 - Geometrical measurement error in reflected laser.

Another consideration is the geometrical error with the sensor. Figure 6 shows the geometry. The path length of the laser can be thought of as a radius to a circle. We want to measure the arc length of the circle as the angle changes from the initial position. For the PSD sensor, we are not measuring the arc length, but rather the projection of the arc length onto the sensor. The optical path determines the resolution of the measurement primarily by increasing the displacement on the PSD. The displacement for some angle from the origin of a perpendicular beam can be expressed, for small angles, as:

$$
x=R \sin \theta
$$

This displacement follows a constant path length, $R$. Any angle, $\theta$, will extend the path length beyond R, increasing the displacement. For small angles, this change can be neglected. At larger angles, this produces an overshoot from the actual displacement. While this is strictly a 
geometrical error, the actual geometry will be difficult to calculate and cannot be completely removed. I have calculated the error based on a laser path normally perpendicular to the sensing area:

$$
\Delta x=R \sin \theta^{2} \tan \theta
$$

This shows that an increase in the path length, $R$, increases the error, as does an increase in the measured angle, $\theta$. The path length, with no corrections made, should be minimized as long as the desired resolution is fulfilled.

Any spherical lens of appreciable thickness will produce a distortion known as spherical aberration, shown graphically in Figure 7. This distortion provides additional focusing power at the edges of the lens, moving the focal point closer to the rear surface of the lens. While typically unfavorable, this system can use this aberration advantageously. At larger angles, the geometrical error will increase. Also at larger angles the spherical aberrations worsen, causing the beam to wander towards the optical axis. At some value, the errors could help cancel each other out for at least some angles. Spherical aberration is difficult to model and this analysis was not performed for the system.

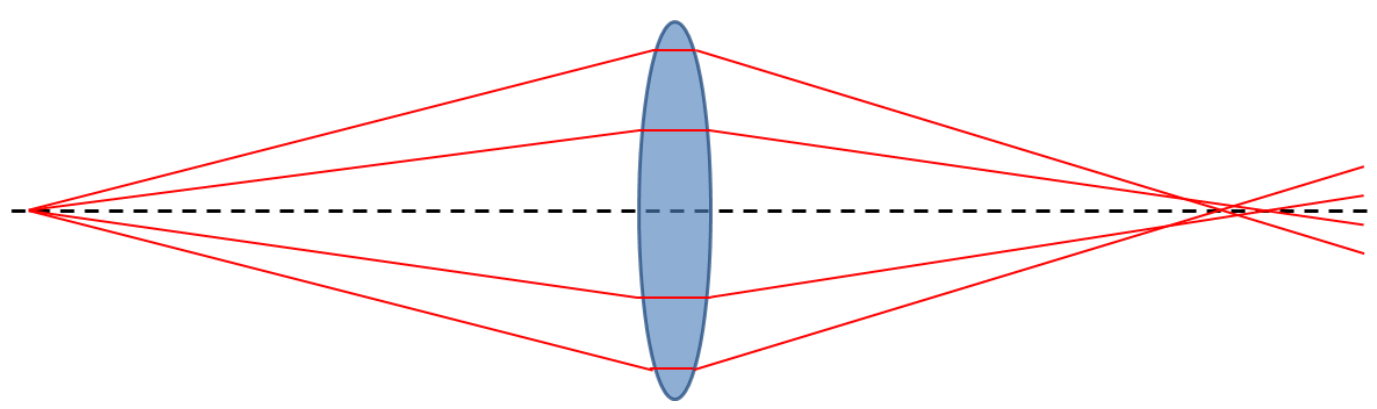

Figure 7 - Spherical aberration occurring in a spherical lens. 


\section{Electronics}

The electronics consist of two components, the signal amplifier and the microcontroller. This was done to keep the size of the optical housing small while keeping the system modular.

The signal amplifier is required to produce an output signal that is compatible with the analog to digital converter (ADC) on the microcontroller. The output from the PSD is a small current, which needs to be amplified and converted to a voltage for the ADC. A transimpedance operational amplifier design was incorporated for this purpose. Figure 8 shows the board layout. The schematic is shown in Appendix B, Figure B2.

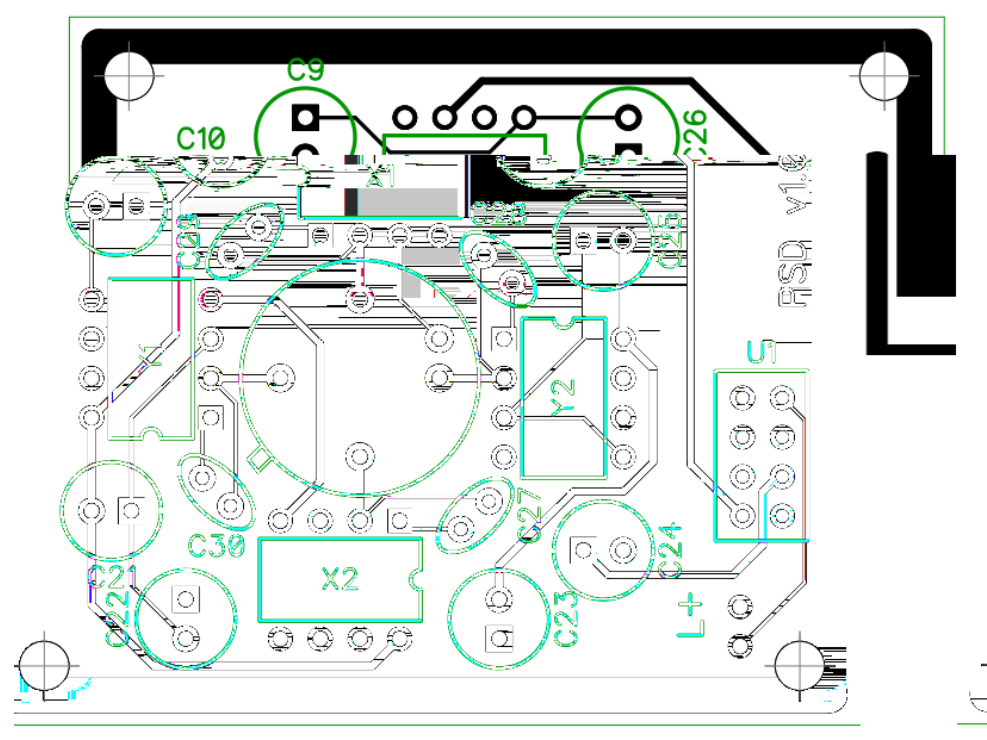

Figure 8 - Amplifier board layout, top layer.

To achieve a high accuracy and low noise, high-quality components were used in the construction of the amplifier. Texas Instruments OPA2132 op-amps were used, and 0.1\% resistors were used for the gain control. The assembled board is shown in Figure 9 below. 


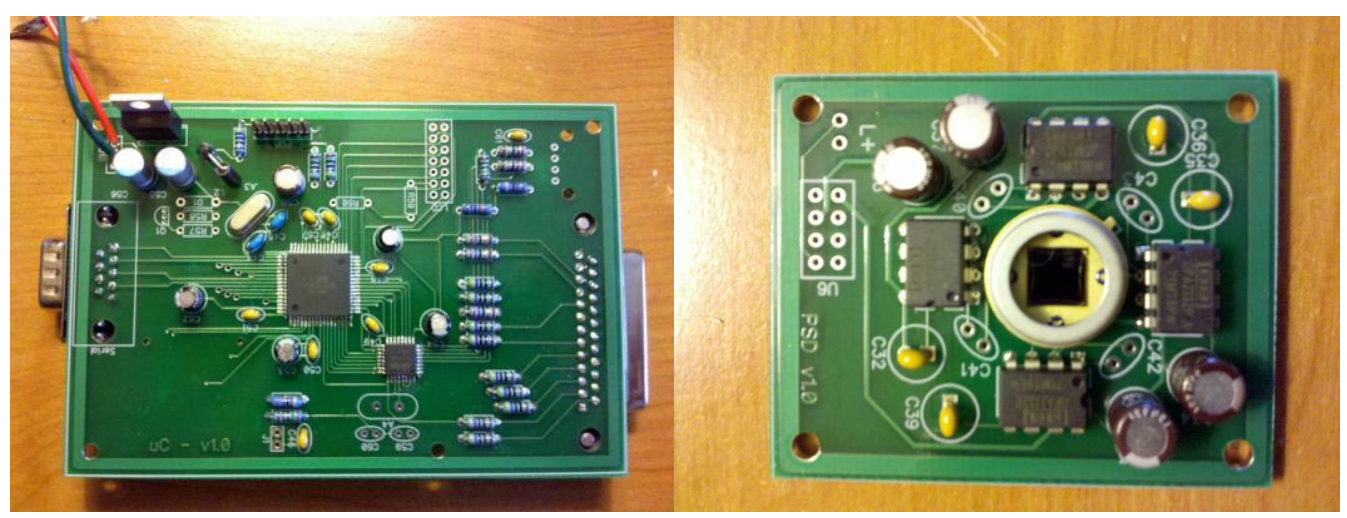

Figure 9 - Assembled circuit boards.

The microcontroller was chosen based on the following factors:

- $\quad$ Fast clock speed (16-32MHz)

- High-resolution, high-speed ADC (12-bit or higher, 20kHz or higher)

- Easy to program

Due to previous experience, the Atmel AVR family of microcontrollers was chosen. From there, the only microcontroller to meet the requirements was the ATxmega.

To communicate with the computer, the parallel port was chosen due to its ease of programming and interfacing. In case it was needed, additional avenues of data transfer were designed in. USB support was added using a parallel-to-USB chip provided by FTDI. A Secure Digital (SD) card slot was added to provide a method of data storage and prevent the need for a computer during measurements. Neither method has been tested or configured.

The ability to use an LCD was designed onto the board primarily for debugging and initial testing before computer software was written. Writing to the LCD screen takes far too long to be used when collecting data, but can be used at a slower speed to test functionality.

The ability to control the laser brightness was added to the board by utilizing the digital-to-analog converter (DAC) on the microcontroller and a field-effect transistor (FET). By controlling the voltage to the FET, more or less current is allowed to pass to the laser diode, controlling its brightness. The microcontroller board is shown in Figure 10. The schematic is shown in Appendix B, Figure B2. 


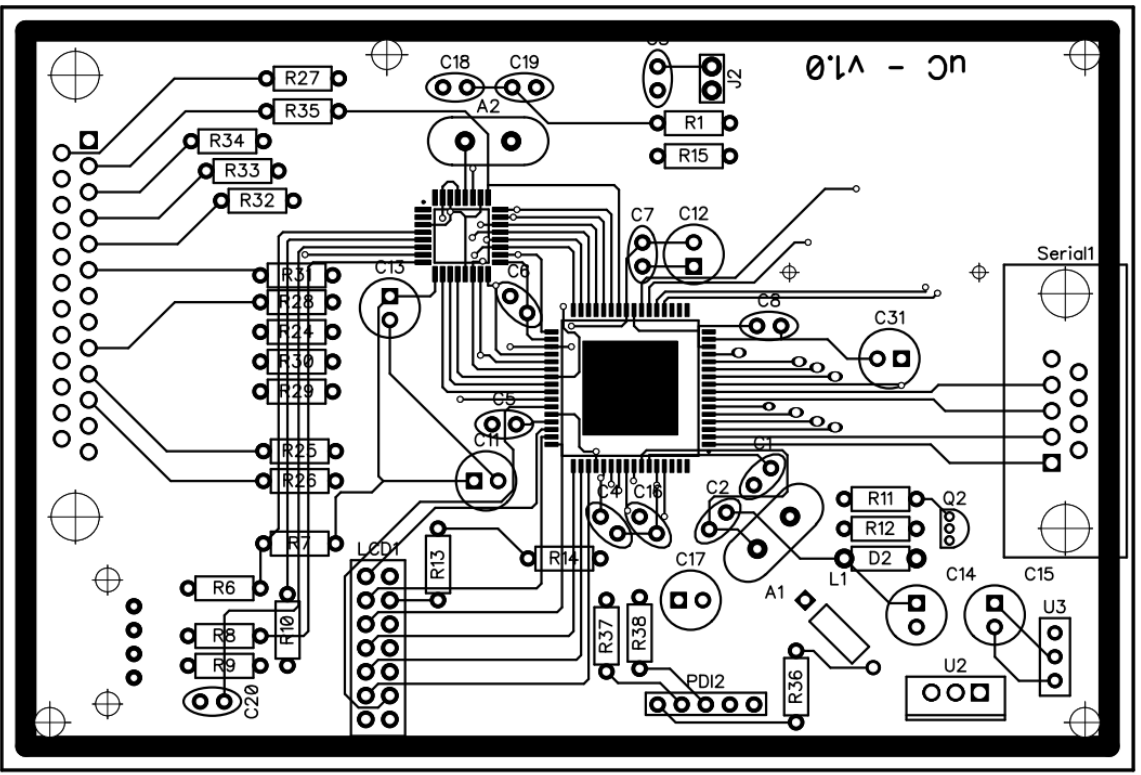

Figure 10 - Microcontroller board layout, top layer.

\section{Software}

Two pieces of software were necessary for this project. The microcontroller required control software for the processing of the collected data, and the PC required software to collect the data.

The microcontroller performs one main task. It is to sample the voltage at each input, calculate the position of the laser, and send that position to the PC via the parallel port. It does this as fast as it can to achieve the highest sampling rate possible. The built in event system of the microcontroller was used to provide consistent and automatic sampling of the ADC. Additional functionality was added to allow the PC software to control several features of the microcontroller.

The microcontroller, upon calculating the 32-bit position of the laser, sends four groups of 8 bits over the parallel port. Two bits on the parallel port's status register uniquely identify each byte of data. There is no flow control built in, and any data lost is not buffered and is not resent. This was done to ensure maximum data transmission and to prevent complication. Figure 11 below shows the general flow of the software. 


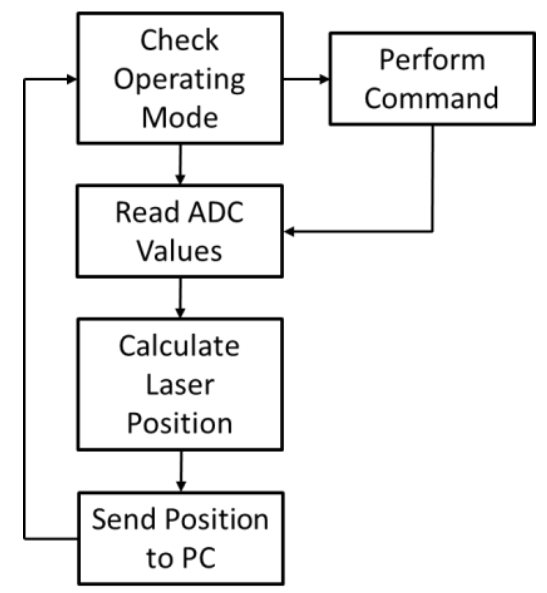

Figure 11 - Microcontroller software flow chart.

The PC software was written to allow data to be recorded by the computer in a way that could easily be imported into Microsoft Excel for manipulation and post-processing. It does this by saving a comma-separated data file (CSD) for importing. Additional functionality was added including a graphical display of the laser position, and provisions for controlling the microcontroller in several key areas. These areas include operating frequency and laser brightness. A screenshot of the software is shown below in Figure 12.

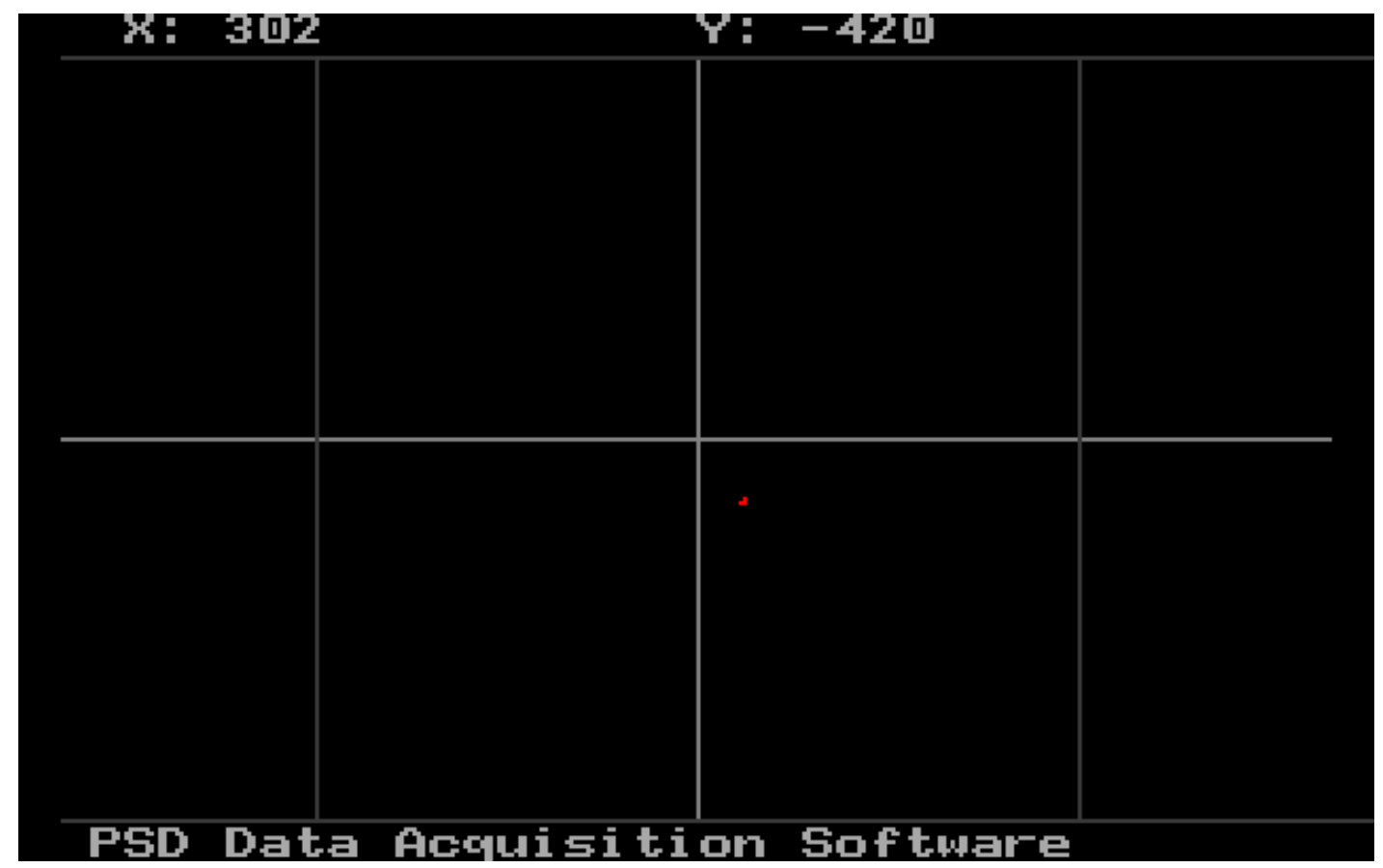

Figure 12 - Data acquisition software screenshot from the PC. 
The computer software polls the parallel port for data by constantly checking the status bits in the status register. Once a change has been detected, data is stored into a temporary buffer. This is done to prevent recording the same data more than once. After both bytes have been received for either the $\mathrm{X}$ or $\mathrm{Y}$ coordinate, the full 16-bit value is stored in the position sample buffer to be written to a file. Figure 13 shows the flow of the computer software.

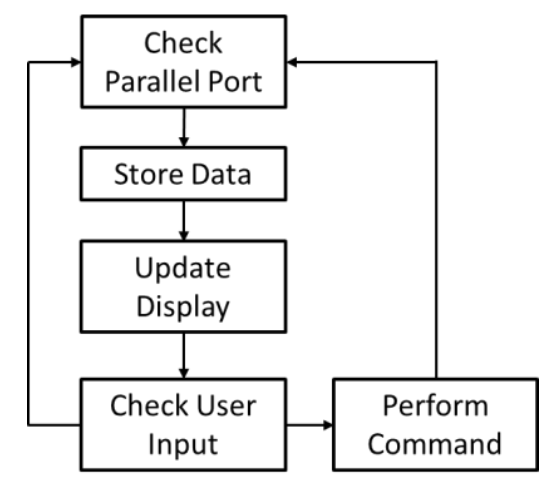

Figure 13 - Simplified software flow chart for PC code.

For usability, the computer software, when not recording data, collects and averages the samples to be displayed in the graphical interface. At this time, it is also possible to press function keys on the keyboard to control settings on the microcontroller. Table 2 shows the function keys. Pressing a function key sends data out the parallel port to be read by the microcontroller. A third bit in the status register controls whether the microcontroller is sending or receiving data. Pressing the sample button runs separate code that prevents any interference to the software by keystrokes and disables the graphical interface. This helps prevent excess code from tying up processor resources and losing valuable data from missed status changes. 
Table 2 - Software function keys.

\begin{tabular}{|c|c|c|}
\hline Offsets & $\mathrm{O}, \mathrm{P}$ & $\begin{array}{l}\text { The offset keys control the ADC value offsets, which affect laser position } \\
\text { calculations. Enabling offsets takes the last ADC readings and stores them } \\
\text { in an offsets buffer. These offsets are then subtracted from every } \\
\text { subsequent ADC reading. This allows for any ambient laser light to be } \\
\text { subtracted from the measurement so that only reflected light influences the } \\
\text { readings. Pressing "P" sets all offsets to 0. Setting the offsets while the } \\
\text { laser is reflected onto the PSD will cause integers to overflow once the } \\
\text { laser light is removed or changes position. Remove offsets if this occurs. } \\
\text { Ideally offsets should be set at the laser brightness used for measurement } \\
\text { with no laser light reflecting onto the PSD. }\end{array}$ \\
\hline $\begin{array}{l}\text { Clock } \\
\text { Divider }\end{array}$ & $1-6$ & $\begin{array}{l}\text { The clock divider keys set the operating frequency of the microcontroller, } \\
\text { affecting its sampling rate. Increasing numbers further divide the clock by } \\
2 \text {. For } 32 \mathrm{MHz} \text { operation, press "1". For } 8 \mathrm{MHz} \text { operation, press "3". } \\
\text { Default is } 32 \mathrm{MHz} \text {. }\end{array}$ \\
\hline $\begin{array}{l}\text { Record } \\
\text { Data }\end{array}$ & $\mathrm{R}$ & $\begin{array}{l}\text { Pressing the "R" key will prompt the system to sample and store data to a } \\
\text { file called DATA.PSD. After R is pressed, } 15,000 \text { data points are collected. } \\
\text { At the default speed, the sampling rate is approximately } 15 \mathrm{KHz} \text {. This } \\
\text { corresponds to just over } 1 \text { second. Higher clock dividers increase the total } \\
\text { sampling time, during which no user input will be processed. }\end{array}$ \\
\hline Persistence & $\mathrm{Z}$ & $\begin{array}{l}\text { In Persistence mode, the laser spot is never erased from the screen. In } \\
\text { normal mode, the last } 25 \text { data points are displayed. This allows the user to } \\
\text { see the current location. If the beam path is important, Persistence mode } \\
\text { should be turned on. }\end{array}$ \\
\hline Laser Color & $\mathrm{C}, \mathrm{V}$ & $\begin{array}{l}\text { An extra feature to the software is the ability to change the color of the } \\
\text { laser displayed on the computer's monitor. While limited in usefulness, it } \\
\text { does provide a way to distinguish previous laser positions by cycling } \\
\text { through the colors. In the normal mode, this is purely cosmetic, but in } \\
\text { Persistent mode ("Z"), all previous points are left on the screen and the } \\
\text { colors help to differentiate previous runs. Pressing V will reset the laser } \\
\text { color. }\end{array}$ \\
\hline $\begin{array}{l}\text { Redraw } \\
\text { Screen }\end{array}$ & $\mathrm{X}$ & $\begin{array}{l}\text { The display occasionally will develop a problem or become saturated with } \\
\text { data points. To solve this, you must redraw the screen by pressing the "X" } \\
\text { key. This will restore the display to the original state. This will not affect } \\
\text { any other settings. }\end{array}$ \\
\hline Interrupt & I & $\begin{array}{l}\text { After initial testing of the software using polling of the parallel port, it was } \\
\text { thought that using interrupts may help increase the speed and reduce errors. } \\
\text { At low speeds the interrupt method worked well, but at higher speeds it did } \\
\text { not. Ideally the software should allow the user to choose which method to } \\
\text { use to ensure quality data. This was never implemented due to the } \\
\text { complexity of merging the two methods. Pressing the "I" key will toggle a } \\
\text { variable in the code, but the variable is unused. }\end{array}$ \\
\hline $\begin{array}{l}\text { Display } \\
\text { Sample }\end{array}$ & $\mathrm{D}$ & $\begin{array}{l}\text { Pressing "D" will draw the laser position for all points in the sample last } \\
\text { taken. It applies a color gradient to the points based on the sample position. } \\
\text { This will not affect any other options. It is recommended that the laser be } \\
\text { turned off before using, or current position data will overwrite sample data. } \\
\text { To remove the sample, clear the screen using the "X" key. }\end{array}$ \\
\hline
\end{tabular}




\section{RESULTS}

Large amounts of data were gathered for this project before any final hardware was built through various testing setups to provide proof of concept milestones. The figures to follow are the most important data sets, showing that the method works and can be adapted for this particular use.

Figure 14 below shows the results of the initial PSD test data. The results show a repeatable, linear relationship between the change in position of the PSD and the orientation of the measurement surface. The test was performed twice using the disk surface for maximum reflection. The equation of the line determines the sensitivity of the setup. With the equation shown, it is approximately $1000 \mu \mathrm{m} /{ }^{\circ}$, producing a resolution (without noise) of $0.001^{\circ}$.

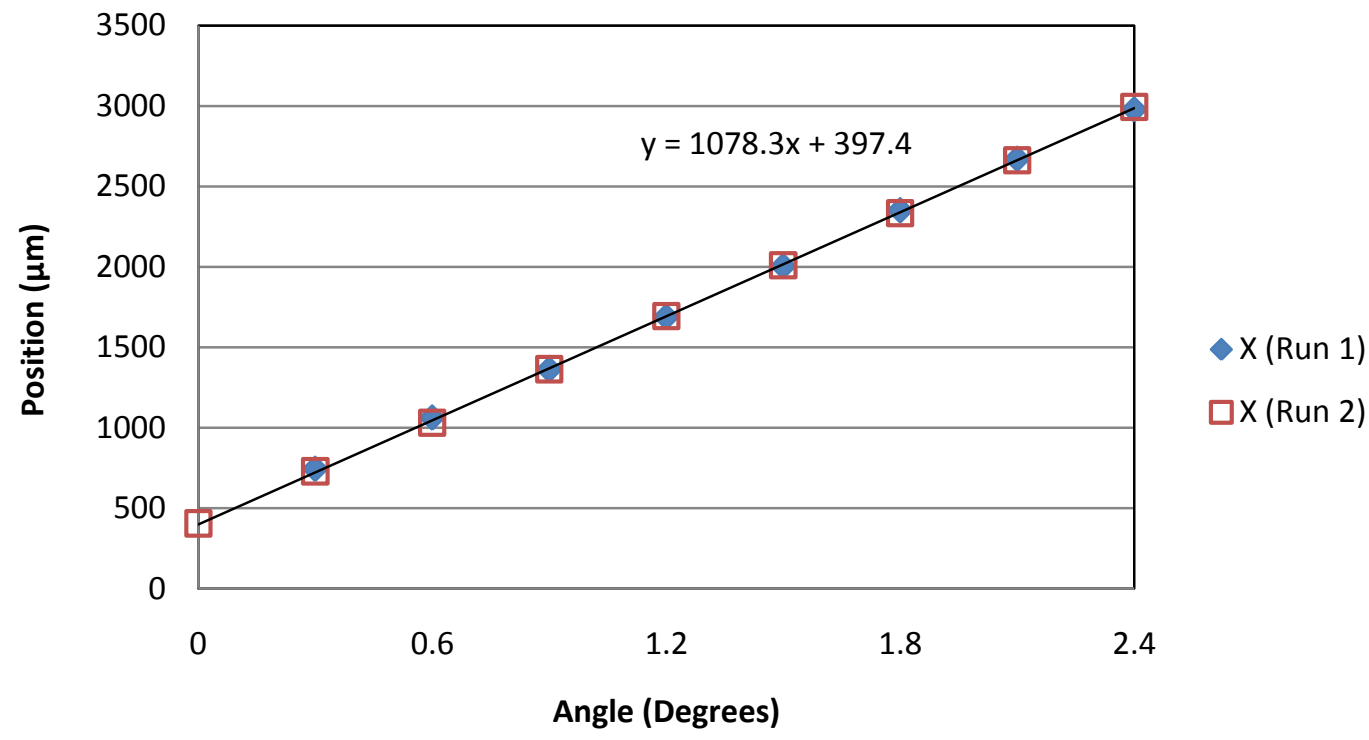

Figure 14 - One-Dimensional PSD Linearity Test.

With the testing method validated, the same test setup was used on the actual two-dimensional PSD. Figure 15 below shows the results of the test. A separate run for $\mathrm{X}$ and $\mathrm{Y}$ was performed. This time the disk surface was used along with a $25.4 \mathrm{~mm}$ focal length lens. As shown, a large amount of spherical aberration can be seen in both $\mathrm{X}$ and $\mathrm{Y}$. The discrepancy between $\mathrm{X}$ and $\mathrm{Y}$ is most likely caused by a misalignment of the lens, causing a greater amount of aberration. Figure 
16 shows the same plot with data gathered using a $50 \mathrm{~mm}$ focal length lens. The spherical aberration is greatly reduced.

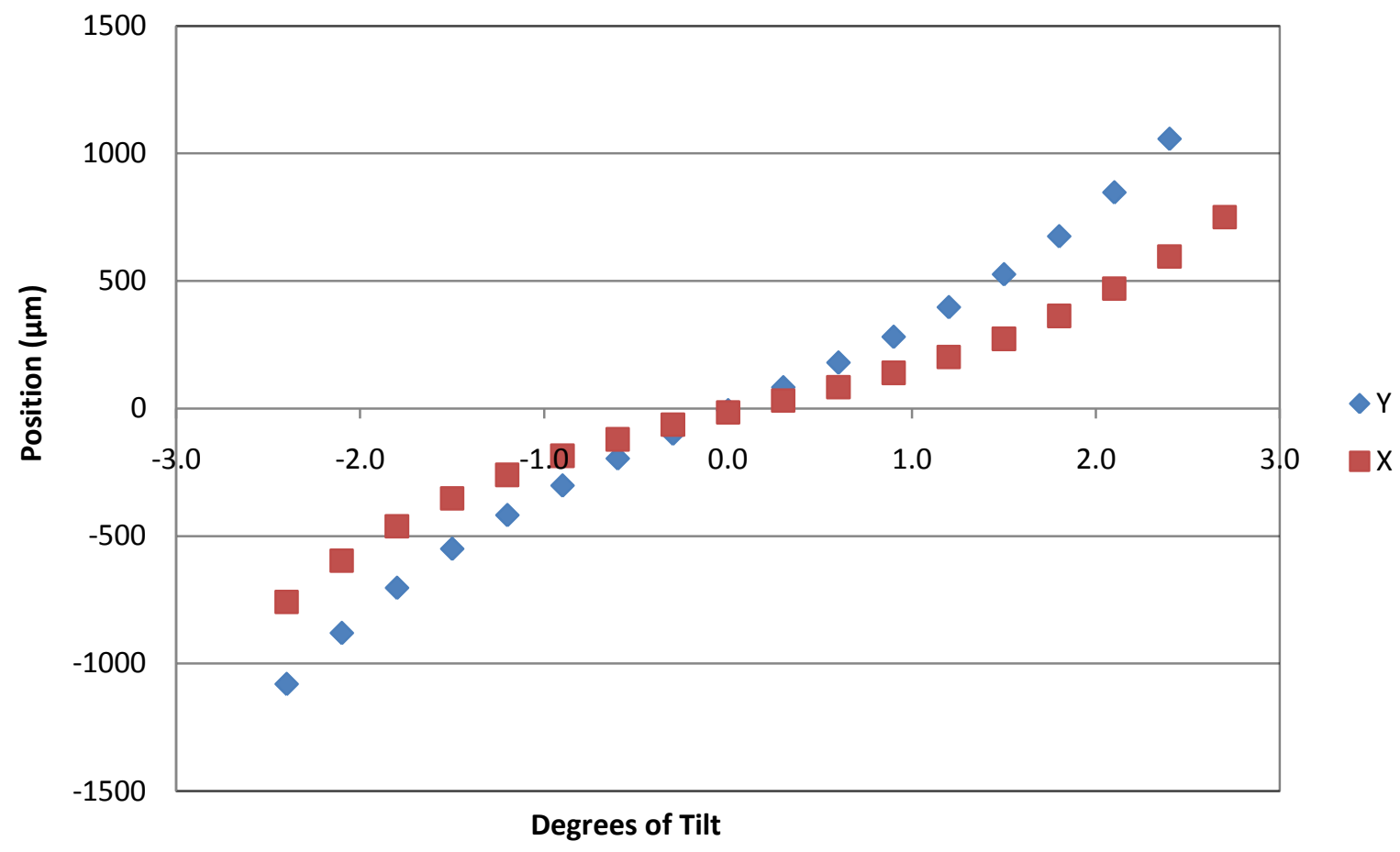

Figure 15 - Two-Dimensional PSD output showing spherical abberation of the focusing lens.

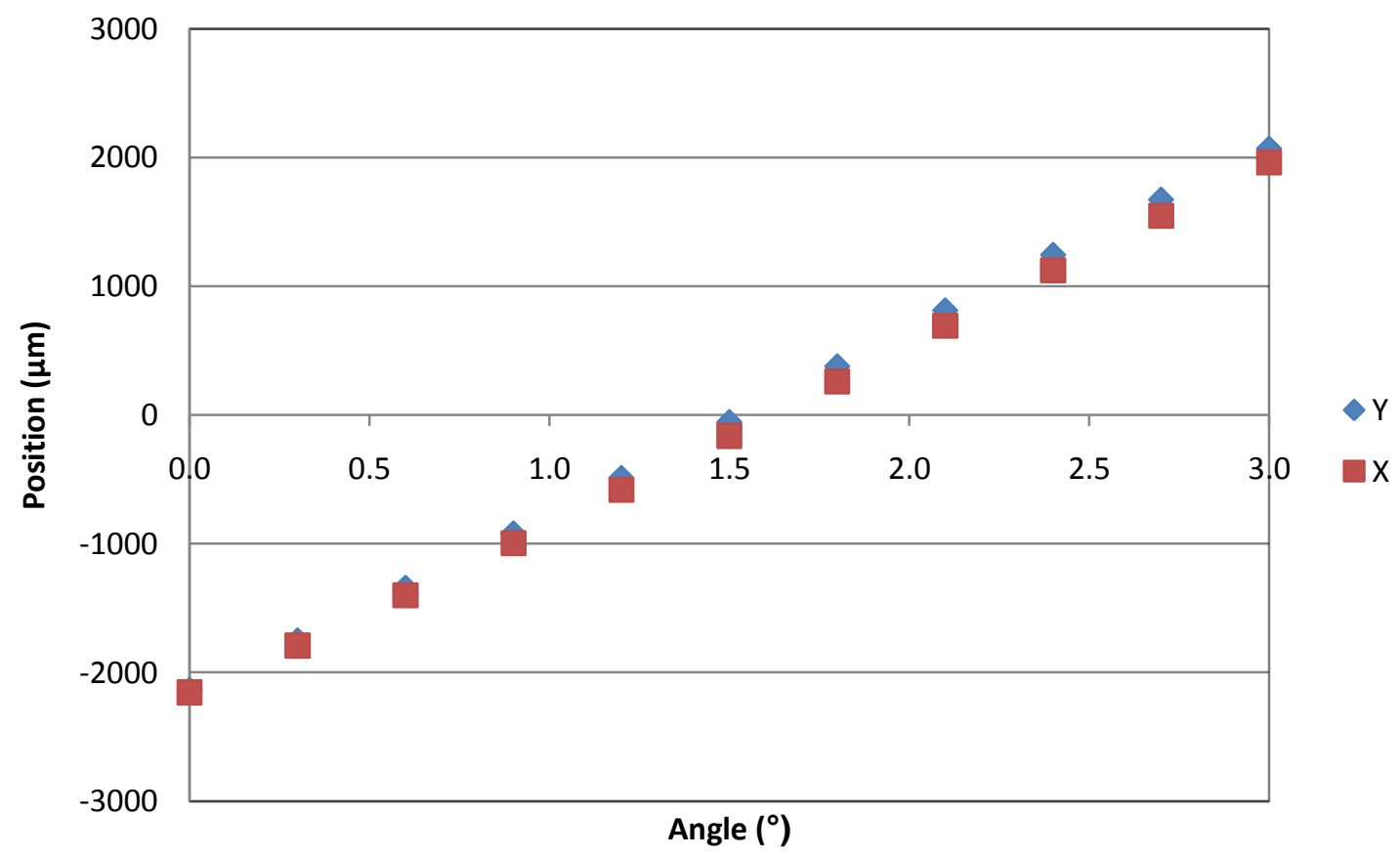

Figure 16 - Validation of the measurement method using the disk surface on the 2D PSD. 
With promising results on the disk surface, the next step was to test the method using the slider surface, as this is the surface of interest. This test was performed in the same manner as the previous test, except using the slider surface and a $35 \mathrm{~mm}$ focal length lens to reduce the spherical aberration. The data is presented in Figure 17. A clear correlation between position and angle can be produced. Low reflectivity caused a much reduced measurement resolution, and the small surface made it extremely difficult to test.

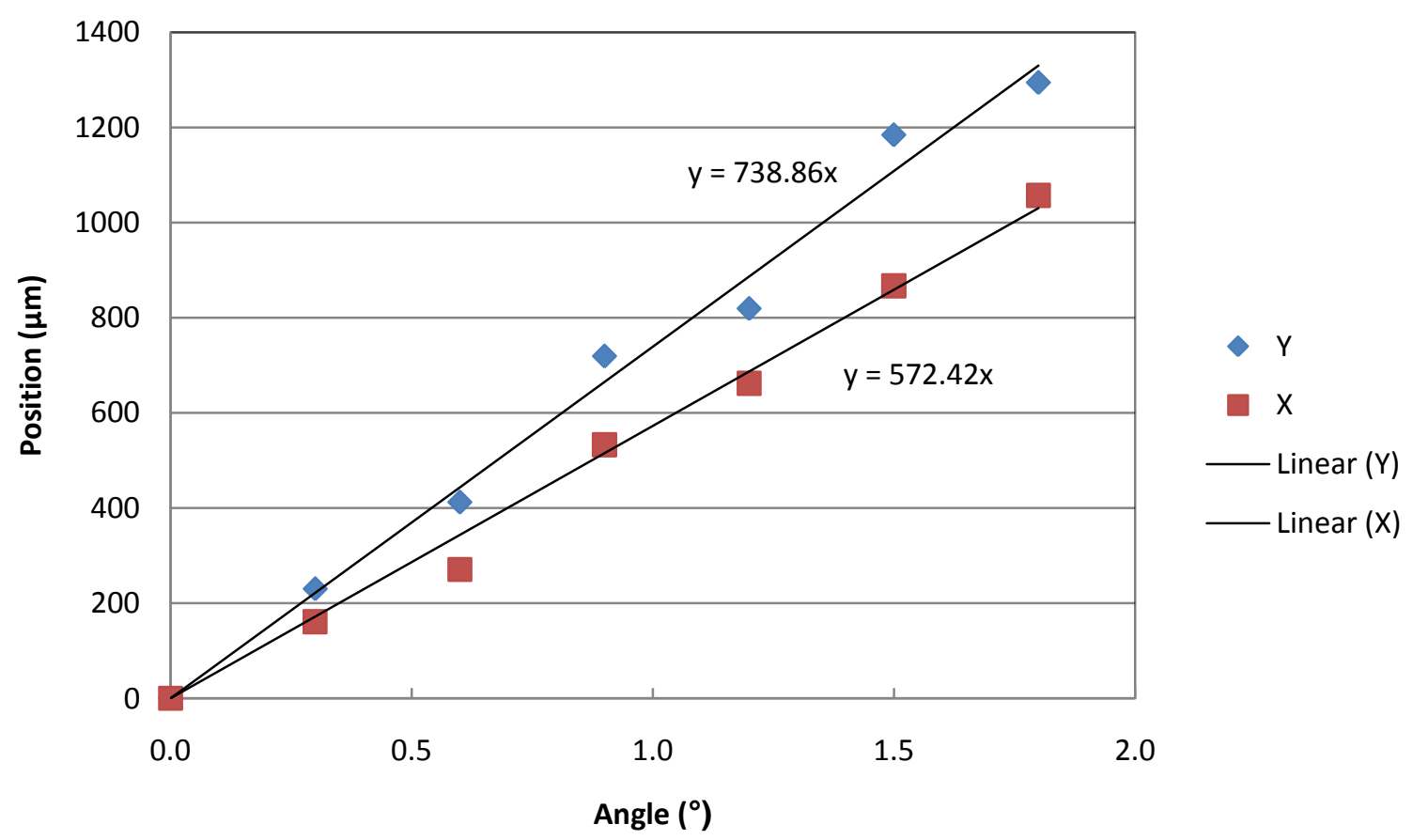

Figure 17 - Validation of the measurement method on the slider surface on the 2D PSD.

The measurement method using reflected light responds to both changes in orientation, which is what is desired, and proximity. In addition, the sensitivity to changes in orientation is affected by the proximity of the sensor to the surface. In order to provide accurate results, the sensitivity must be determined at a variety of distances so that a function could be developed to determine the measured angle. Figure 18 shows the results of the data. Although an offset is present from either misalignment or some other cause, the slopes of the lines are very close, affirming a linear relationship between sensitivity and proximity. 


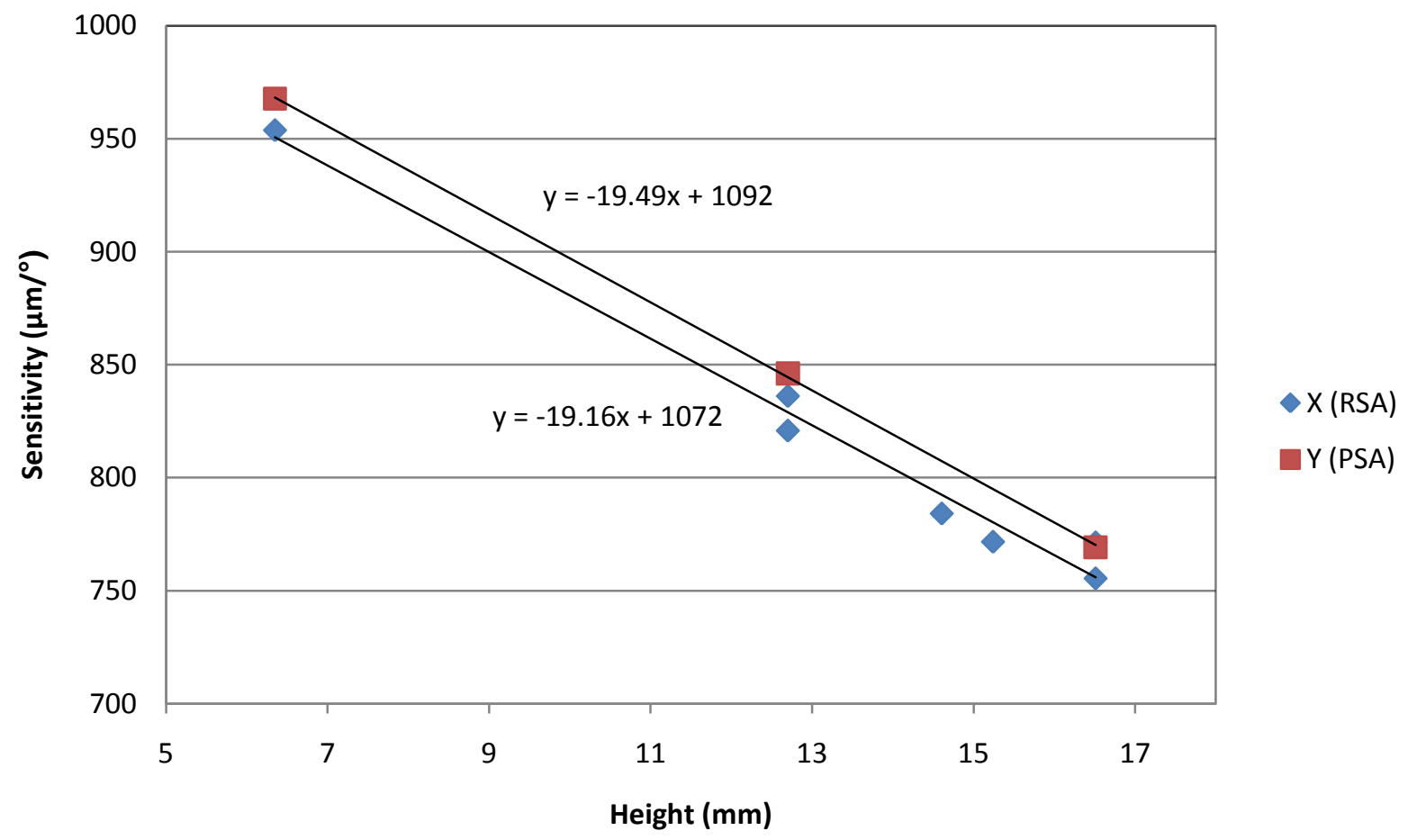

Figure 18 - Measurement system sensitivity as a function of proximity to the measurement surface.

Once the system was characterized, the last step was to get the data acquisition working. In this mode, the microcontroller is sampling and processing as many samples as it can every second. The computer constantly checks whether data is available to collect. Using a pin on the microcontroller that toggles after every sample, the sampling rate was measured to be approximately $15 \mathrm{KHz}$. Figure 19 and Figure 20 below show data collected from the disk surface and the slider surface, respectively. Both graphs have a running average filter applied to reduce noise. Figure 21 shows data measured from a still surface. The output remains flat, and some noise and spurious points are visible. This shows that the measurement method is stable, and that there are relatively few data transmission errors in the data acquisition system. 


\section{Spinning Disk Measurement \\ 20 Sample Running Average}

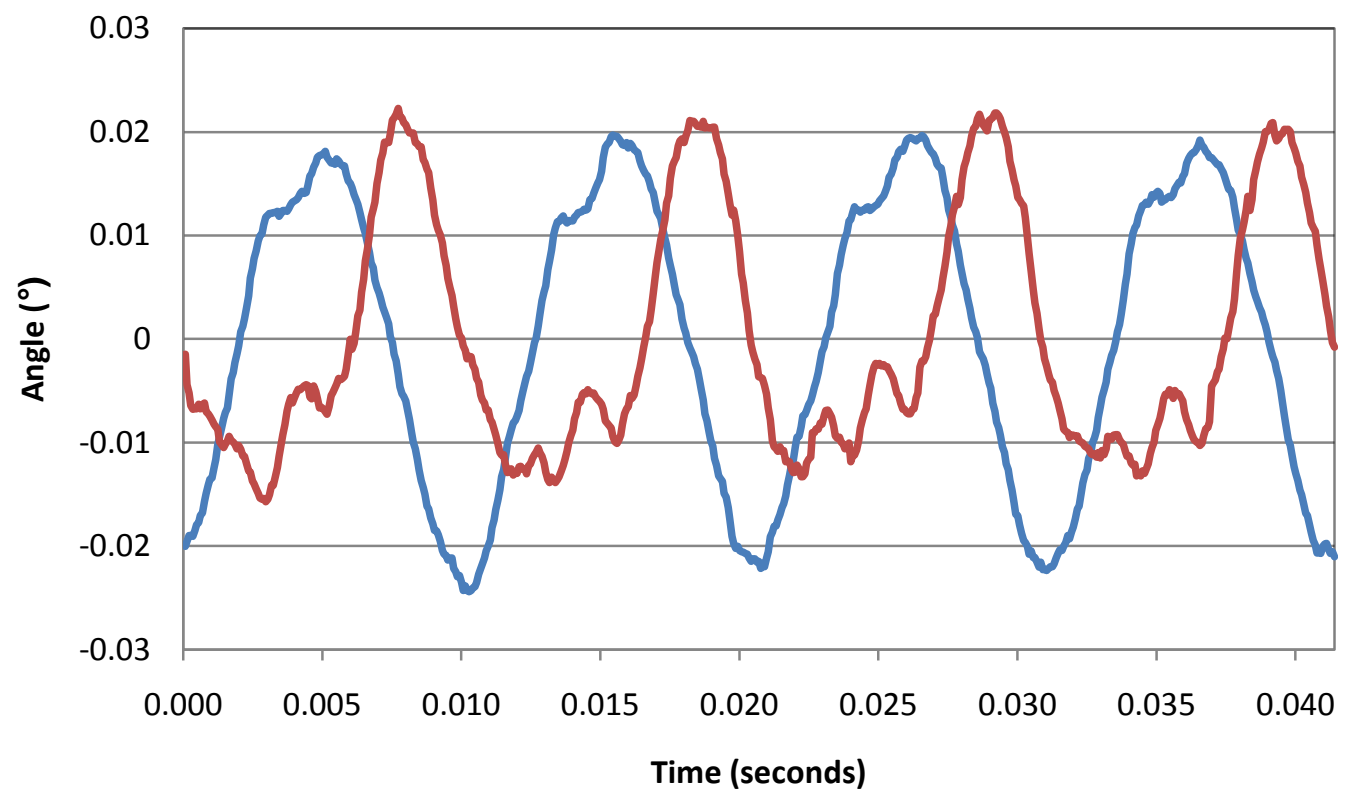

Figure 19 - Measurement system output from the disk surface, with averaging.

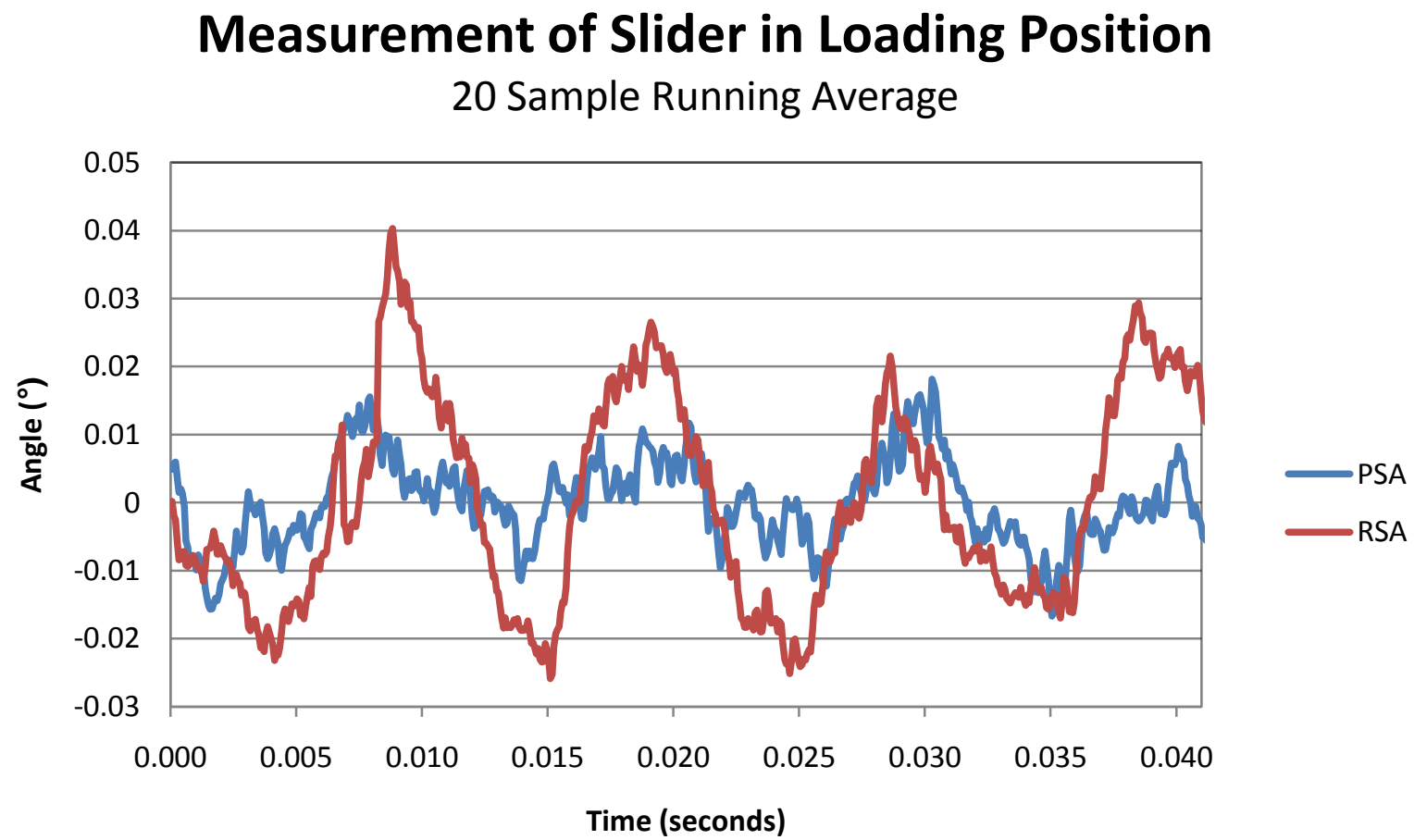

Figure 20 - Measurement system output from the slider surface, with averaging. 


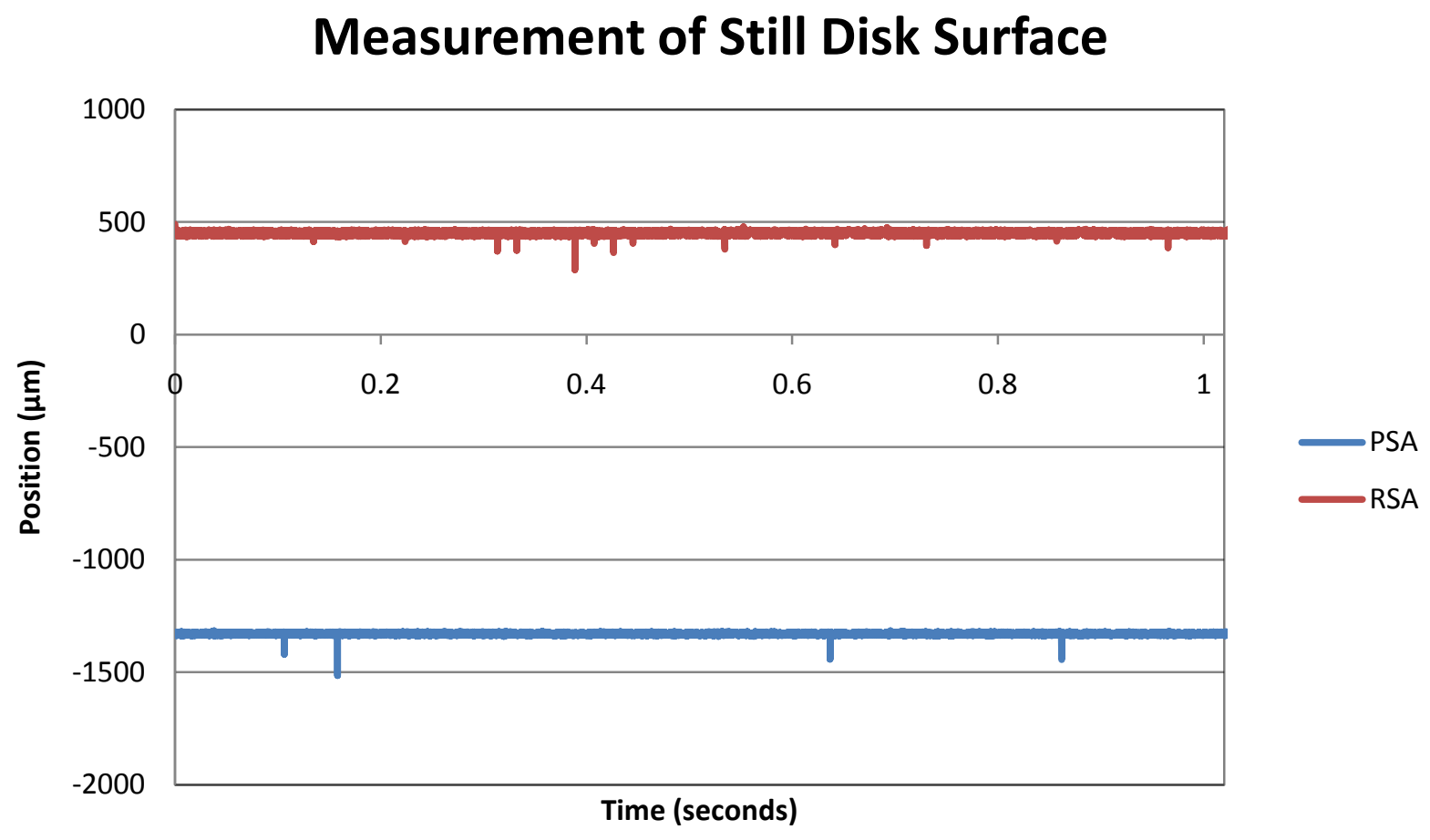

Figure 21 - Still disk surface measurement, showing a constant reading with few errors. 


\section{CONCLUSION}

This project has successfully demonstrated that the PSD measurement method can reliably measure the change in orientation of a slider surface with respect to a reference surface. It has also been shown to work reliably for measuring the orientation of the slider continuously.

Many aspects of the measurement unit can be modified for improved performance. The $5 \mathrm{~mW}$ laser diode appeared faulty and never produced more than $1 \mathrm{~mW}$ of output power when measured. The system was designed for $5 \mathrm{~mW}$ of optical power. Optionally, the signal amplifier could be modified to provide more gain. The laser diode's case is positive and connected to the chassis, while the board was designed to ground to the chassis. Plastic spacers were used to prevent a short circuit.

Due to its simplicity, the parallel port was chosen to transfer the data between the microcontroller and the PC. This presents a concern for newer computers, which typically do not have the legacy ports. The ability to use USB connectivity was designed in but never used. Additionally, an SD card port was designed and built in but never used as well. These could provide additional ways to transfer the data. Additionally, these methods could provide faster data transfer speeds or increase the reliability of the data transfer.

The PC software was written for DOS for its simplicity and ability to read and write directly to hardware. Porting the software to a more modern operating system could improve its usefulness as well as its performance. MATLAB would be an ideal candidate, if the computer's performance was great enough, to serve as the programming platform. Data analysis could be combined using the same software package. However, at the time the software was written, MATLAB does not provide a way to enable bi-directional support for the parallel port, preventing the microcontroller from communicating with the PC. Other data transfer methods could enable MATLAB compatibility. 
In its current state, the software and data are usable. To improve performance and reliability, several aspects could be improved. Each position is truly a 12-bit number, unless the laser runs off the sensing area. The data therefore could be sent over the parallel port in three bytes instead of four. This could improve performance marginally at the cost of more complicated code to split and reassemble the data. Some type of flow control could be implemented in lieu of speeding up the data transfer.

The required calculations by the microcontroller represent my best ability to optimizing performance by performing the fewest calculations possible. The main performance penalty is the use of long integers, which takes the 8-bit microcontroller much longer to process. Code performance also varies based on the values of the data being processed. To prevent this variable delay, which is easily measured using a frequency counter on Port B pin 1 of the microcontroller, the code should be rewritten in assembly language. This will produce repeatable code regardless of the data being processed, and will most likely result in an overall improvement in processing performance. The $\mathrm{A} / \mathrm{D}$ is triggered automatically using the event system, only the code performance needs improvement for data acquisition.

For a future design revision, the outputs from the amplifiers should have a small filter capacitor right before the microcontroller's ADC. The current system samples at $15 \mathrm{KHz}$, while the amplifiers should be capable of amplifying well into the hundreds of kilohertz. This can cause an effect known as aliasing, causing a low-frequency noise caused by a high-frequency source. This will help prevent high-frequency noise from affecting results, as well as reducing noise picked up in the cabling after the amplifier stage.

In general, the resolution of the system exceeds expectations. During testing, the system was calibrated only with a tilt table that could not exceed an accuracy of $+/-0.01^{\circ}$. With better calibration, the measurement can be improved. 


\section{REFERENCES}

[1] Zhu, Hong and David B. Bogy. "Effects of Pitch Static Attitude and Roll Static Attitude on the Steady Performance of Air Bearing Sliders."Journal of Tribology, Vol. 129. Pp. 689694. 2007.

[2] Yonemura, Shinji, Stefan Weissner, Lin Zhou, Frank E. Talke. "Investigation of disk damage caused during load/unload using a surface reflectance analyzer." Tribology International, Vol. 38, pp. 81-87. 2005.

[3] "Interferometry." Wikipedia, The Free Encyclopedia. 6 Nov 2009. 10 Nov 2009 <http://en.wikipedia.org/w/index.php?title=Interferometry\&oldid=324263287>.

[4] "Plane (geometry)." Wikipedia, The Free Encyclopedia. 4 Nov 2009. 10 Nov 2009 <http://en.wikipedia.org/w/index.php?title=Plane_(geometry)\&oldid=323827335>.

[5] “LK-G5000 Series.” Keyence America. 7 Oct 2009.

[6] “LT-9000 Series Laser Confocal Displacement Meter.” Keyence America. 16 Oct 2009.

[7] Wikipedia contributors. "Charge-coupled device." Wikipedia, The Free Encyclopedia. Wikipedia, The Free Encyclopedia, 26 Sep. 2009. Web. 13 Oct. 2009.

[8] Hamamatsu Corporation. Position Sensitive Diodes."Characteristic and Use."

[9] Vision Systems Design. 18 Oct 2009.

[10] “Optoelectronic Gauging.” Marposs Technologies. 6 Nov 2009. 9 Nov 2009. <http://www.marposs.com/technology.php/eng/optical_gauging>.

[11] "Magnetoresistance." Wikipedia, The Free Encyclopedia. 17 Sep 2009. 9 Nov 2009 $<$ http://en. wikipedia.org/w/index.php?title=Magnetoresistance\&oldid=314545395>

[12] "Capacitive Sensor Operation and Optimization (How Capacitive Sensors Work and How to Use Them Effectively).” Lion Precision. 2009. 10 Nov 2009. 


\section{APPENDIX A - Code \\ Microcontroller Code: WDPSD.c}

The microcontroller code was written using Atmel's AVR Studio software. It is written in standard $\mathrm{C}$ and is compatible with the avr-gcc compiler.

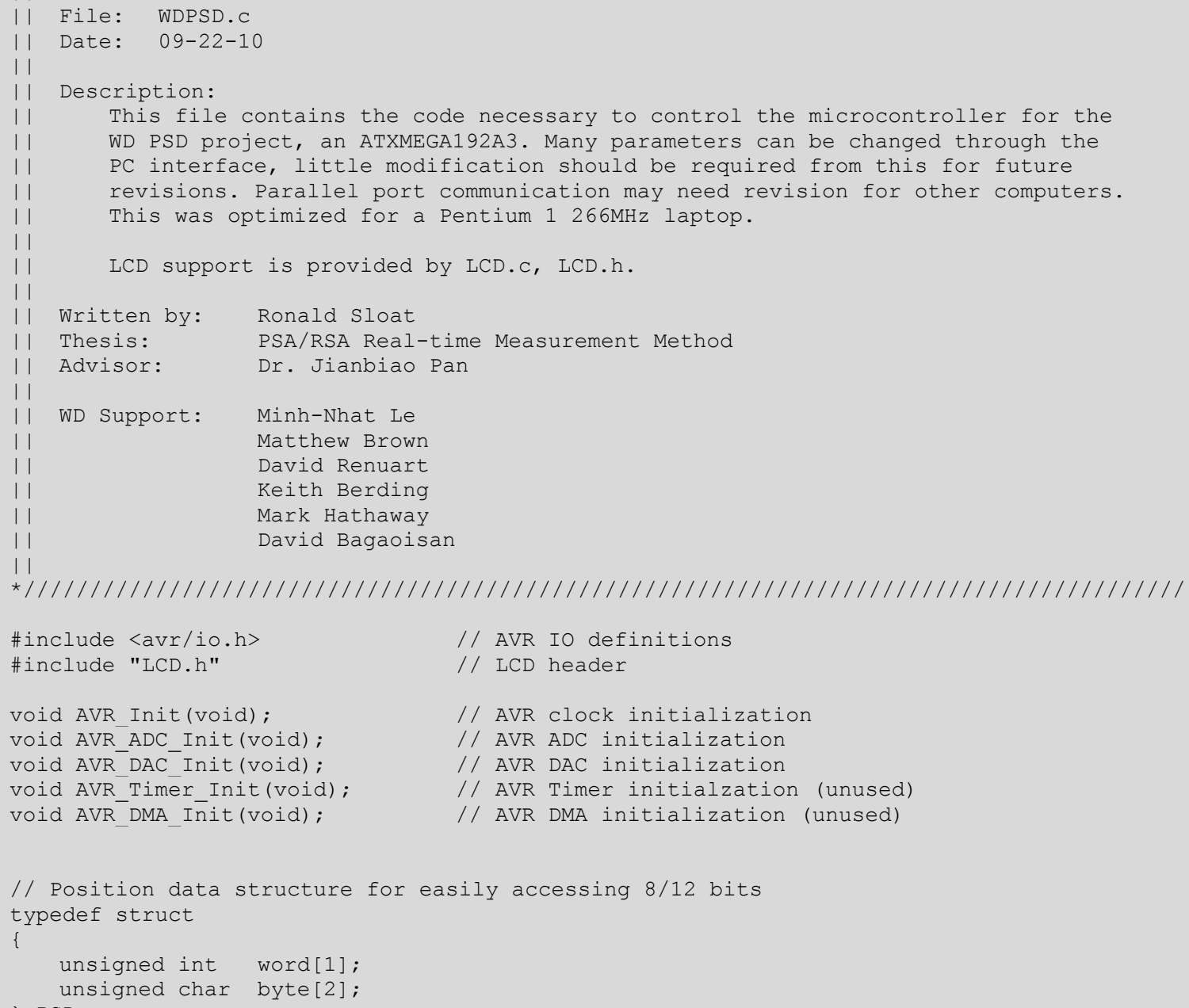




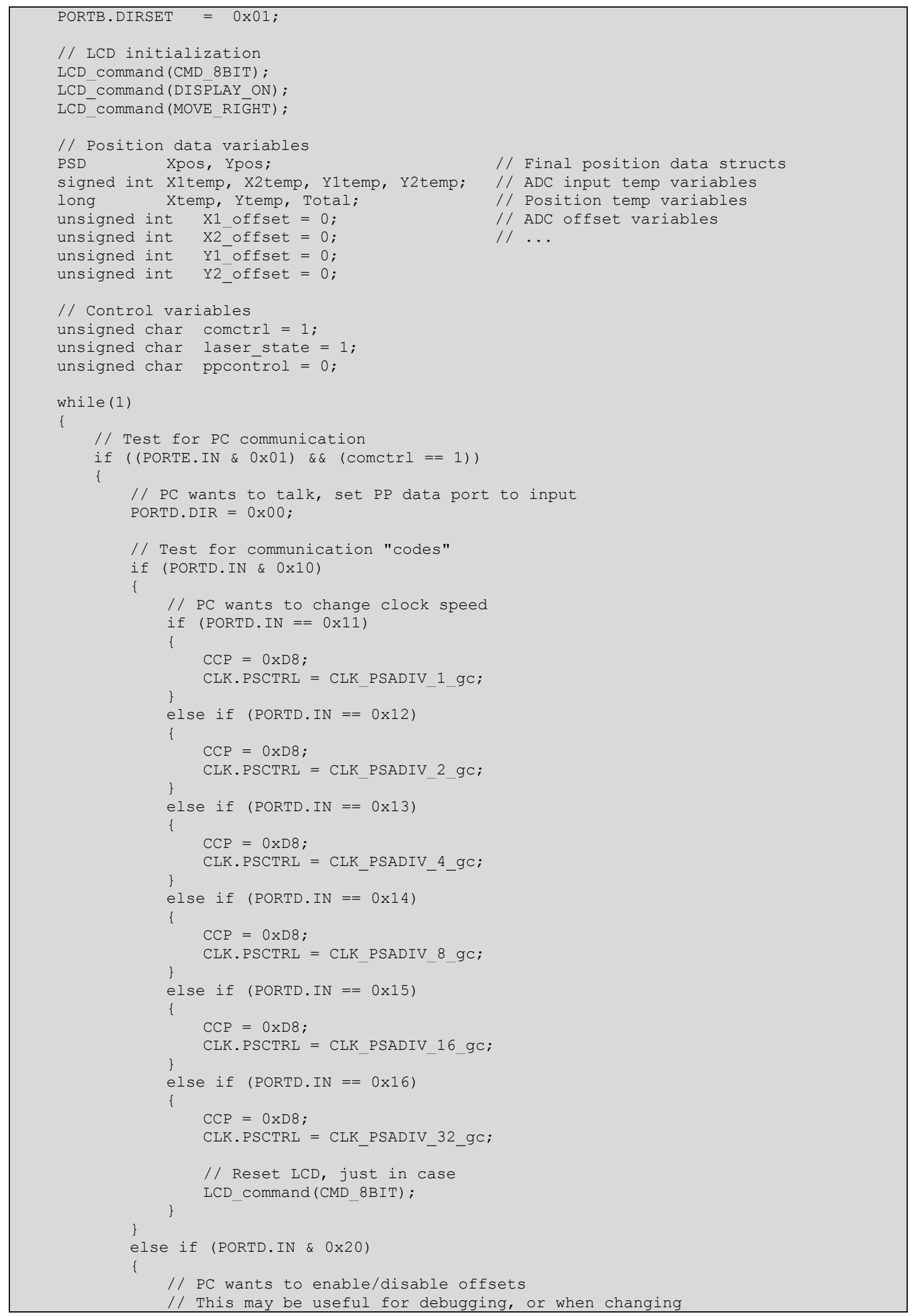




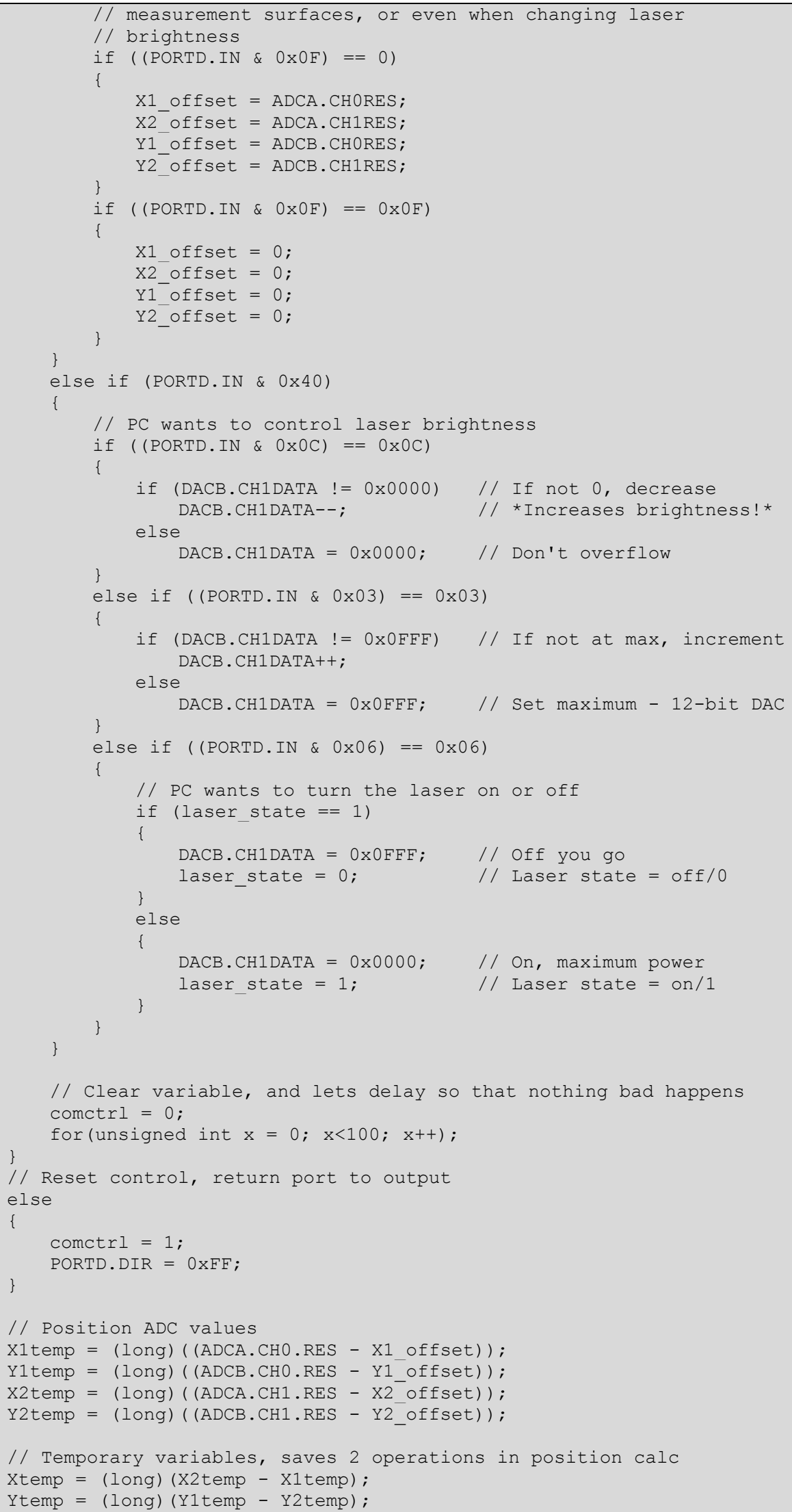




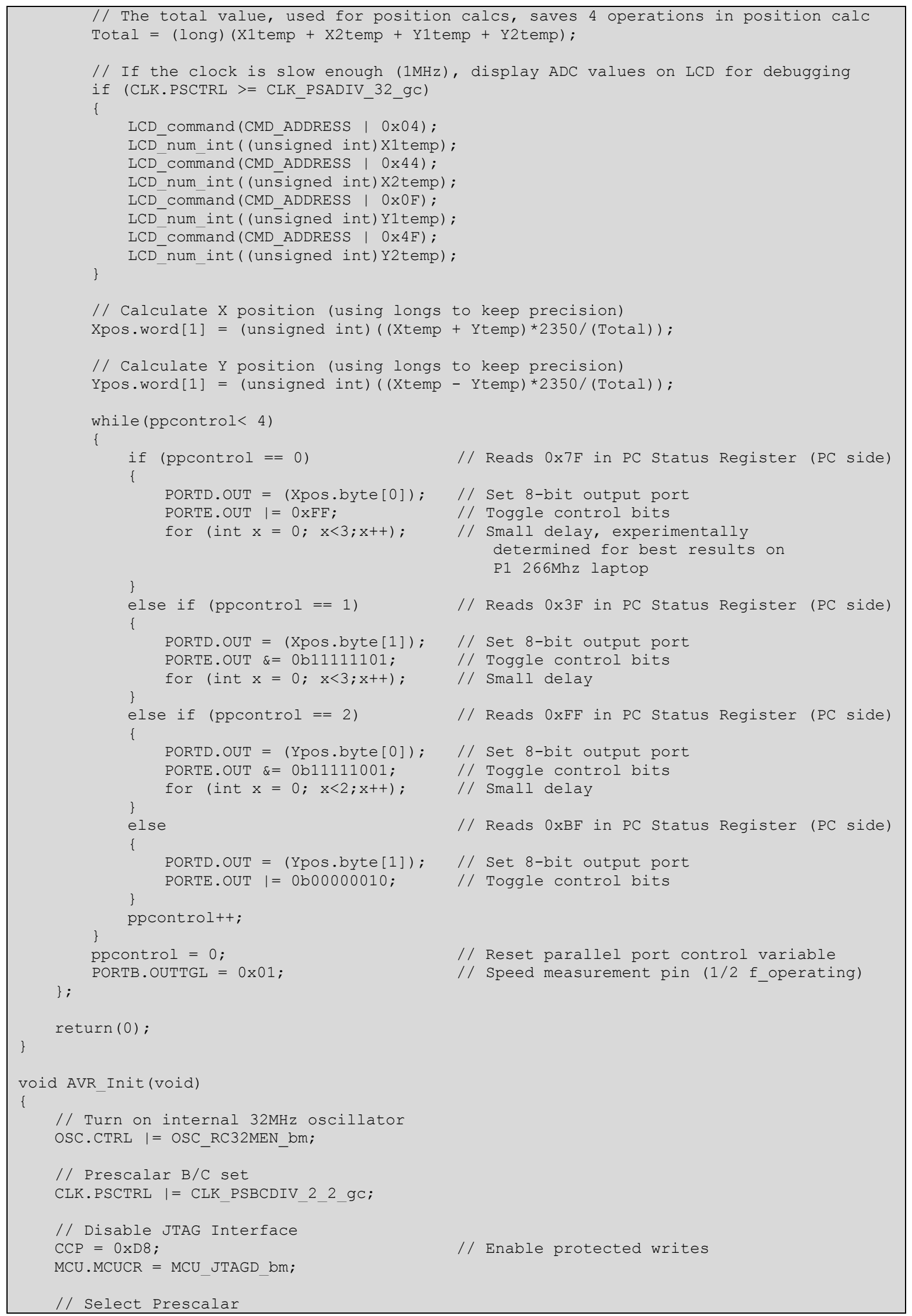




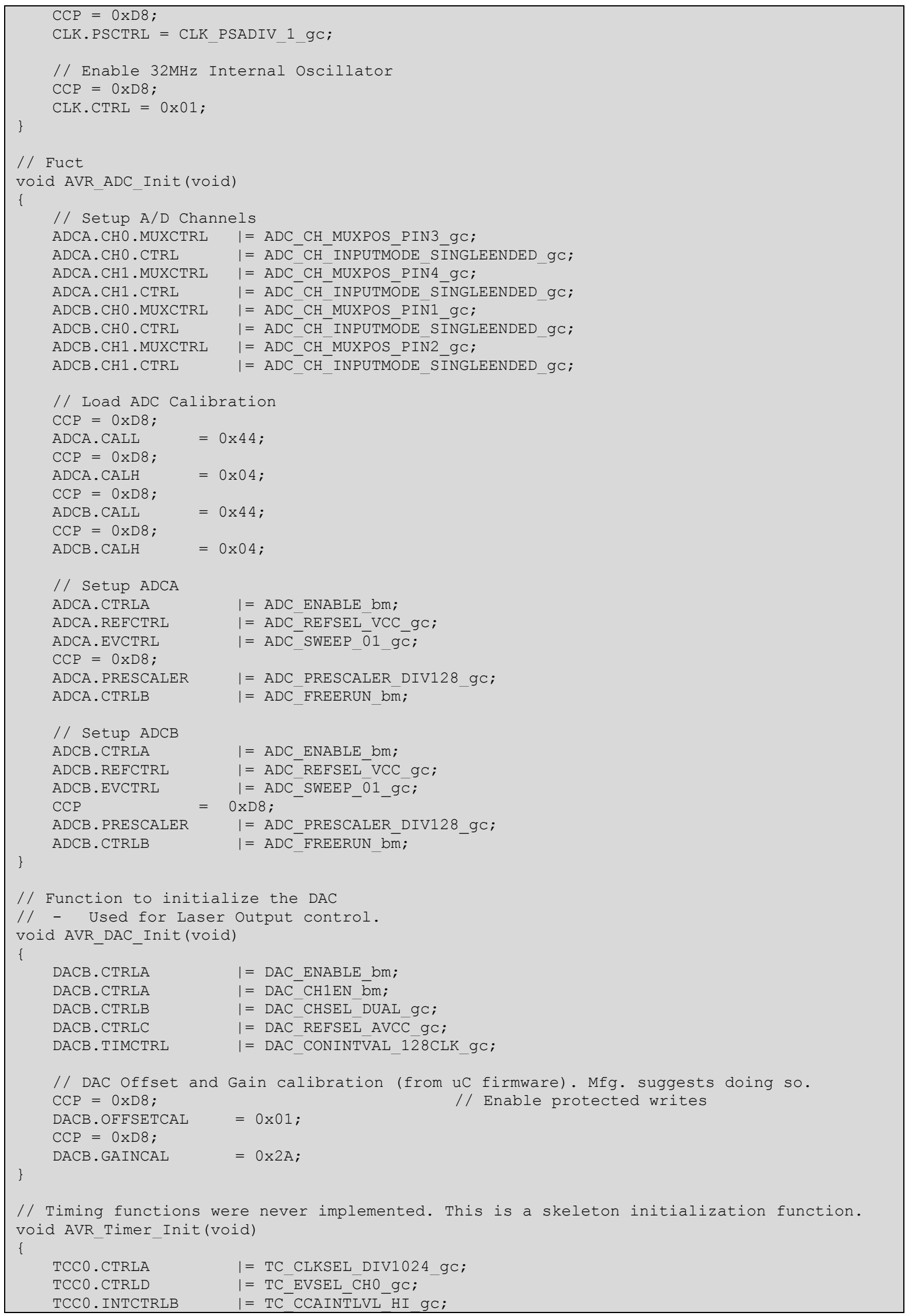




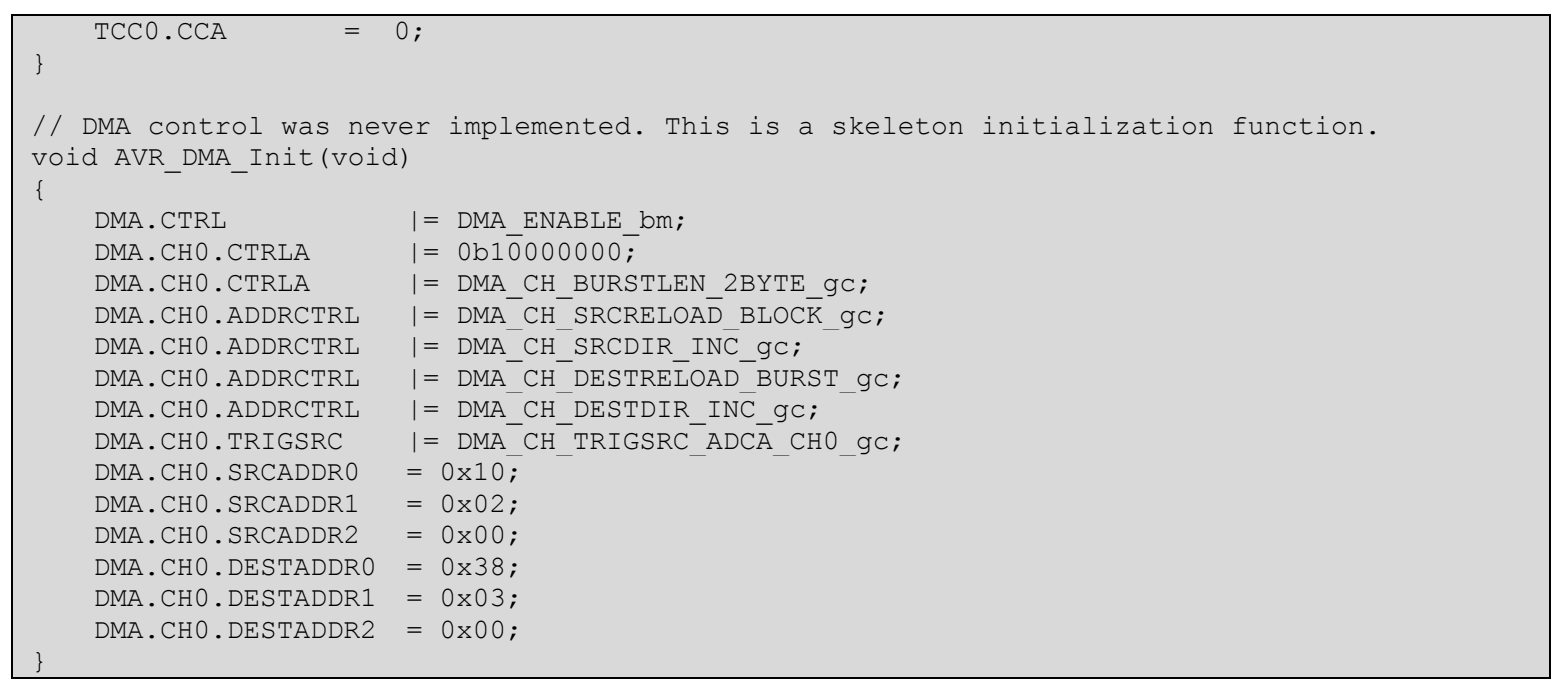




\section{Computer Code: PSDDAQ.CPP}

The computer software was written in Borland C++ 3.1 for DOS. Although it should be mostly compatible with other compilers, it uses inline assembly code that is specific to the Borland compiler. Changes to the inline assembly code will need to be changed if compiling on other software. Additionally, a function library could be used in place of the inline assembly as it is only performing a simple $\mathrm{I} / \mathrm{O}$ read or write. This will most likely slow the software down considerably.

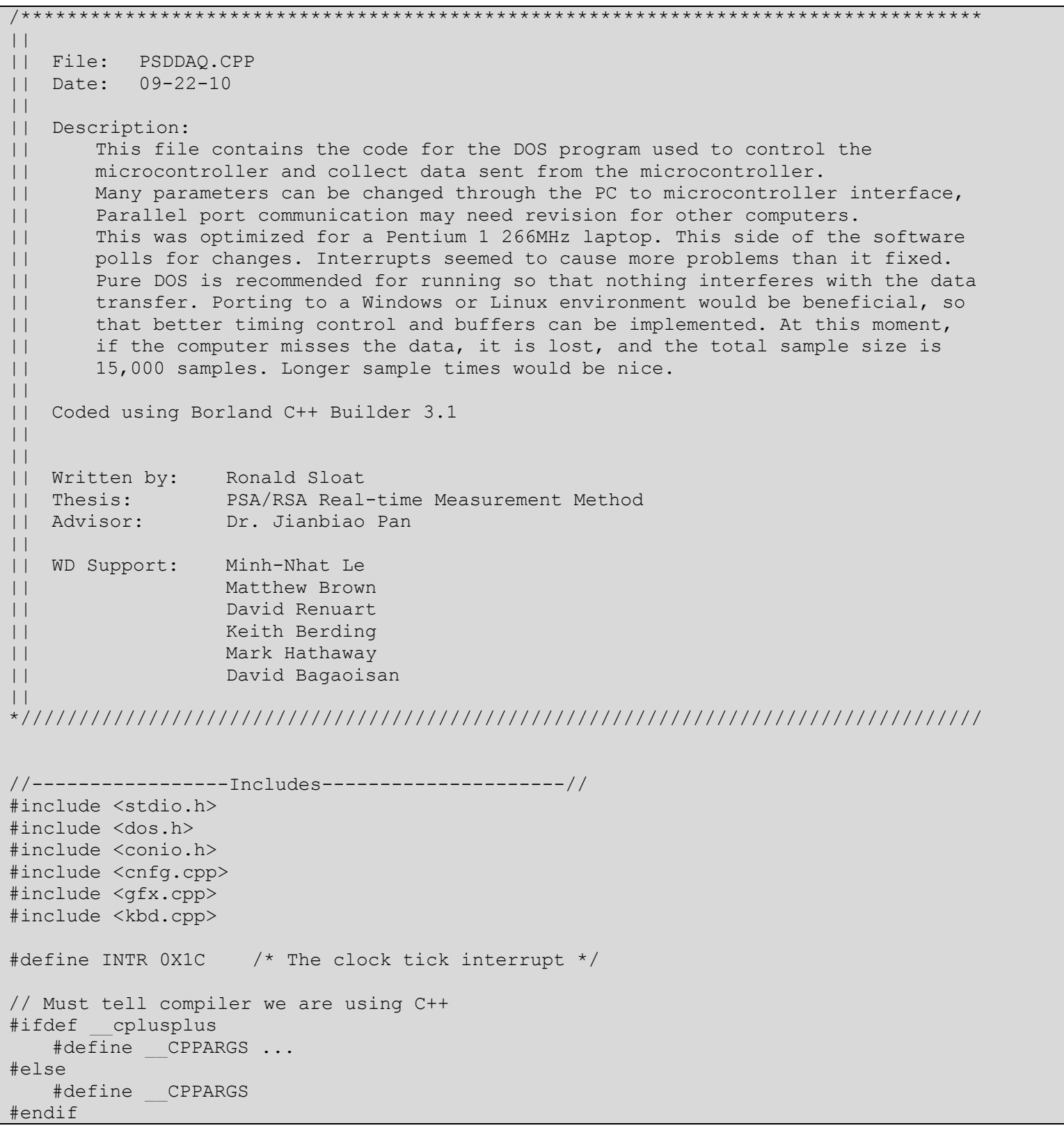




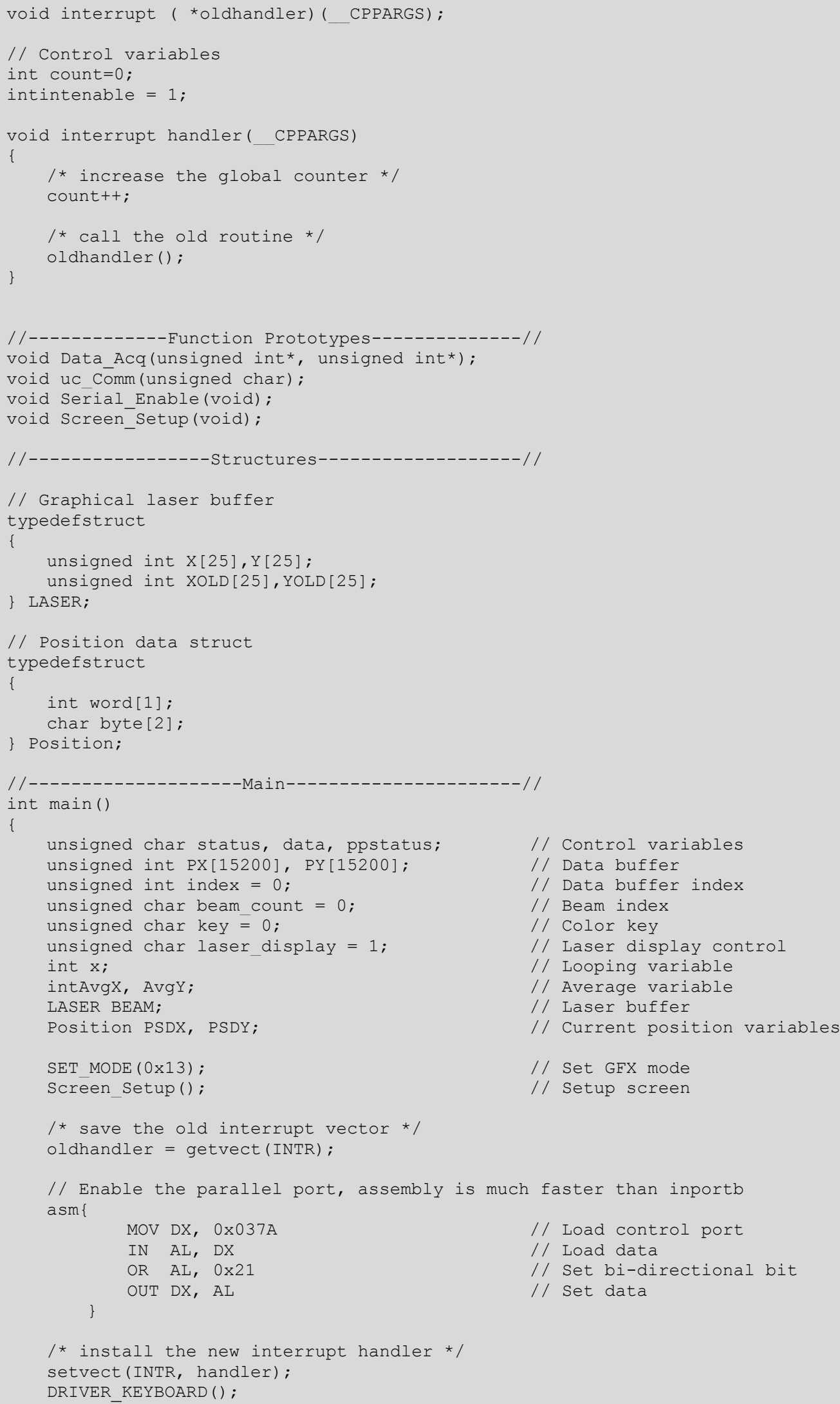




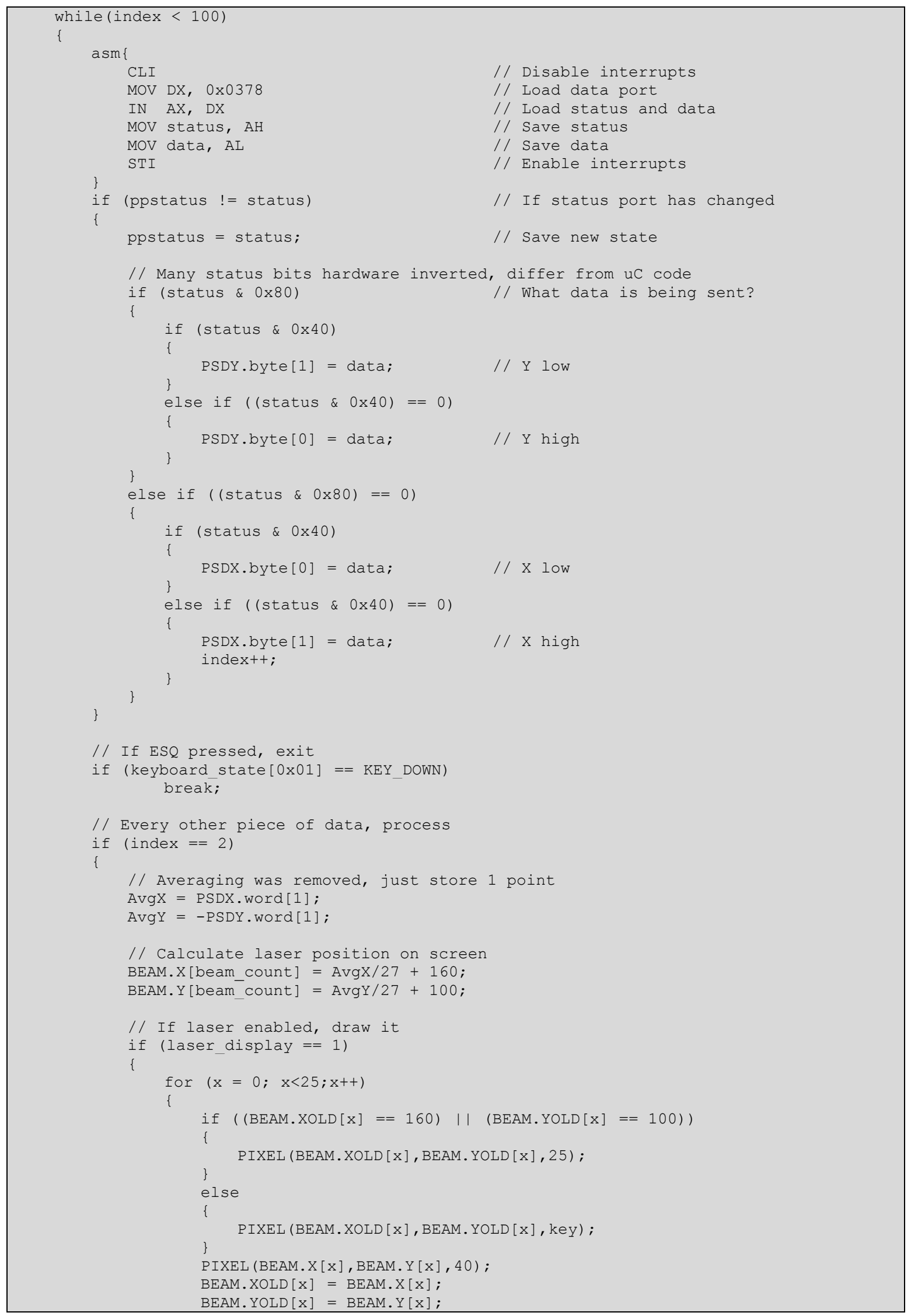




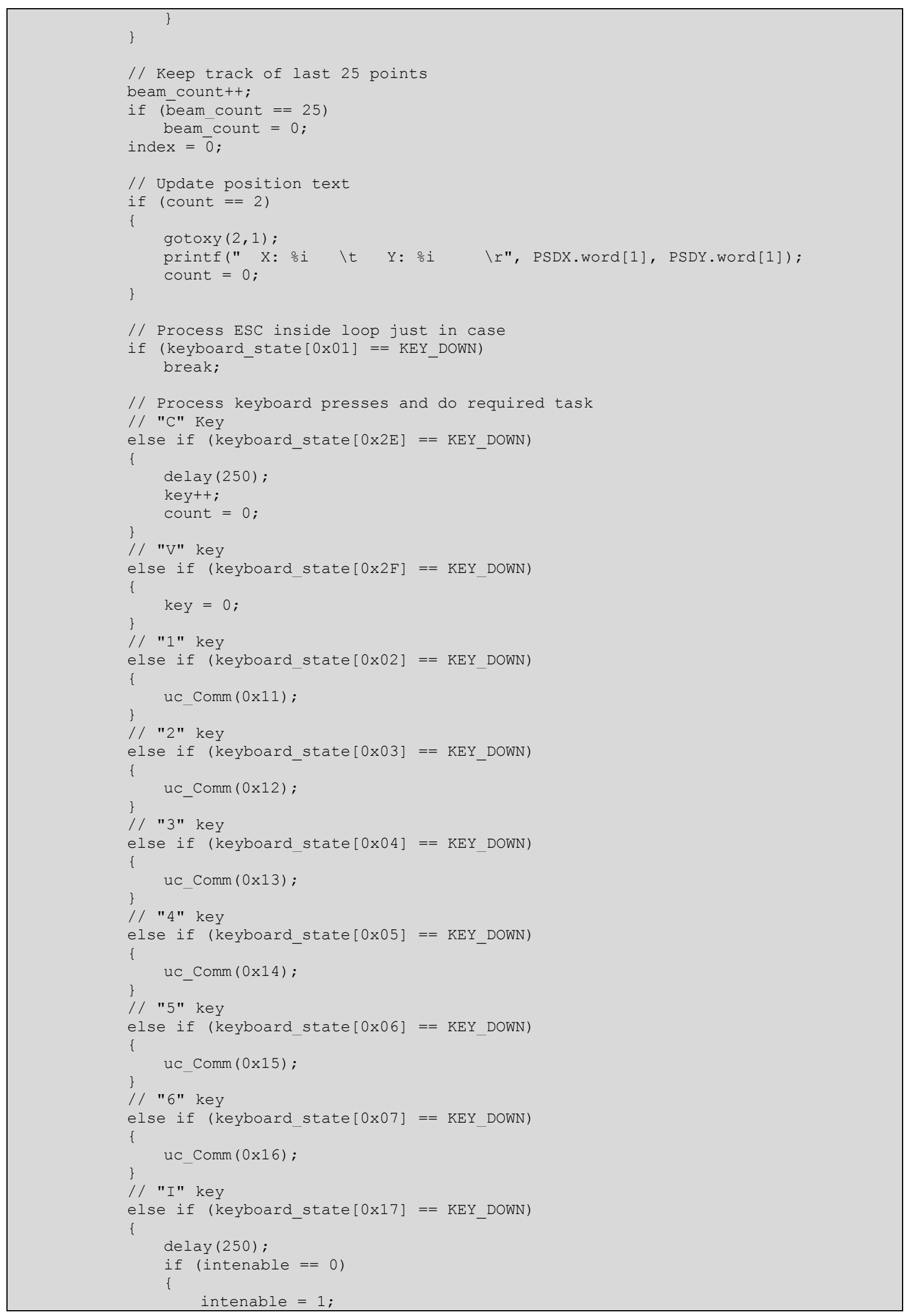




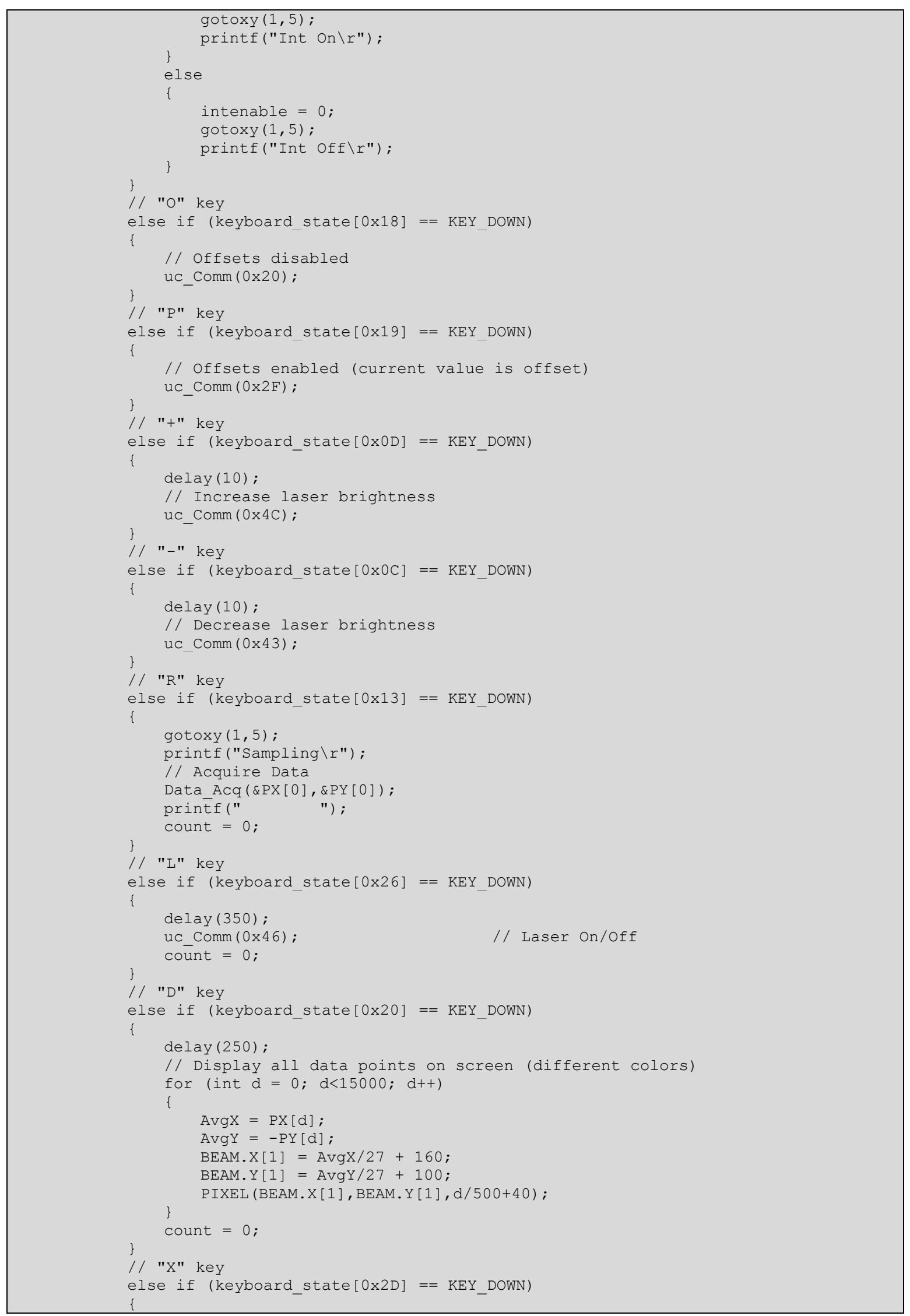




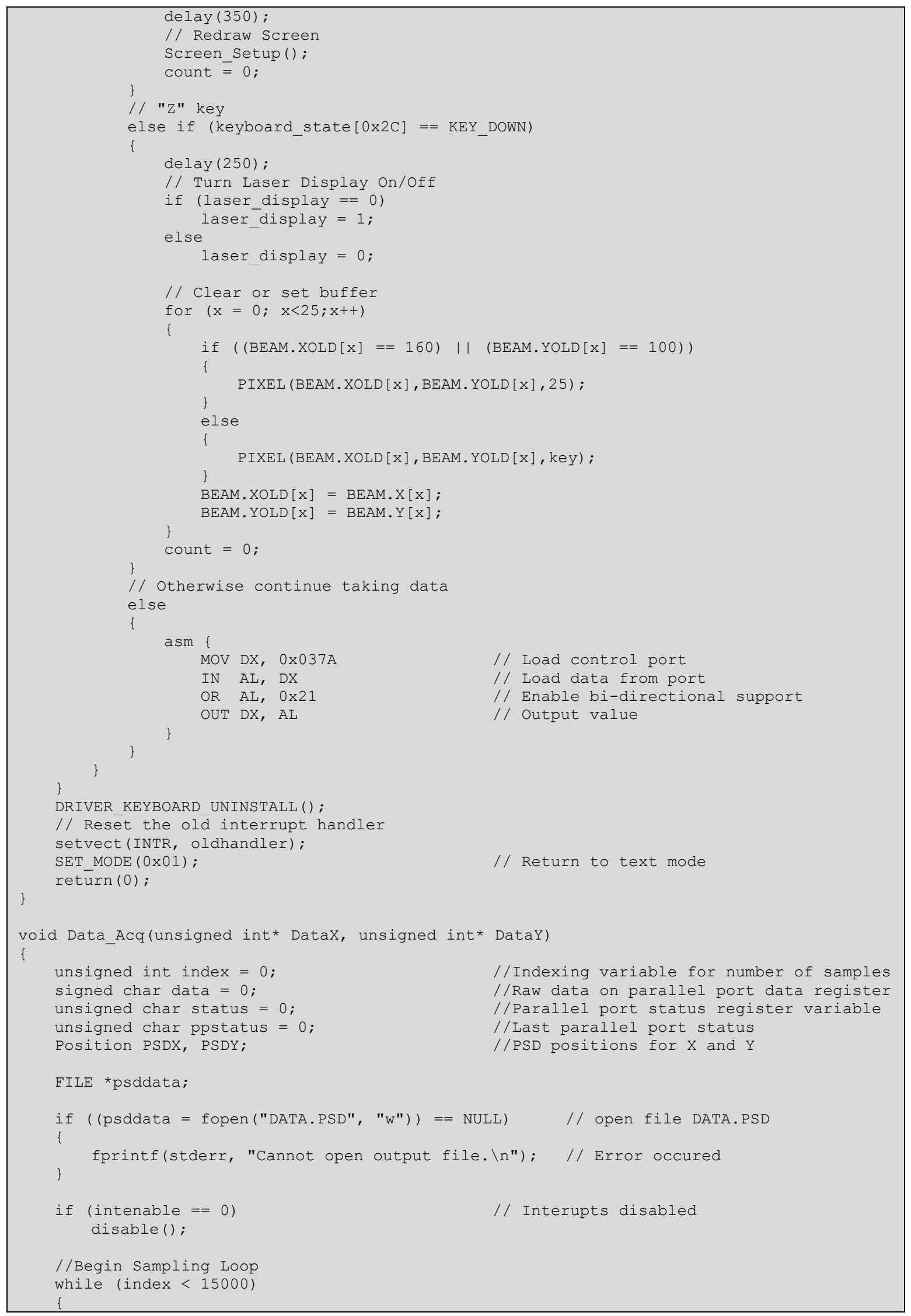




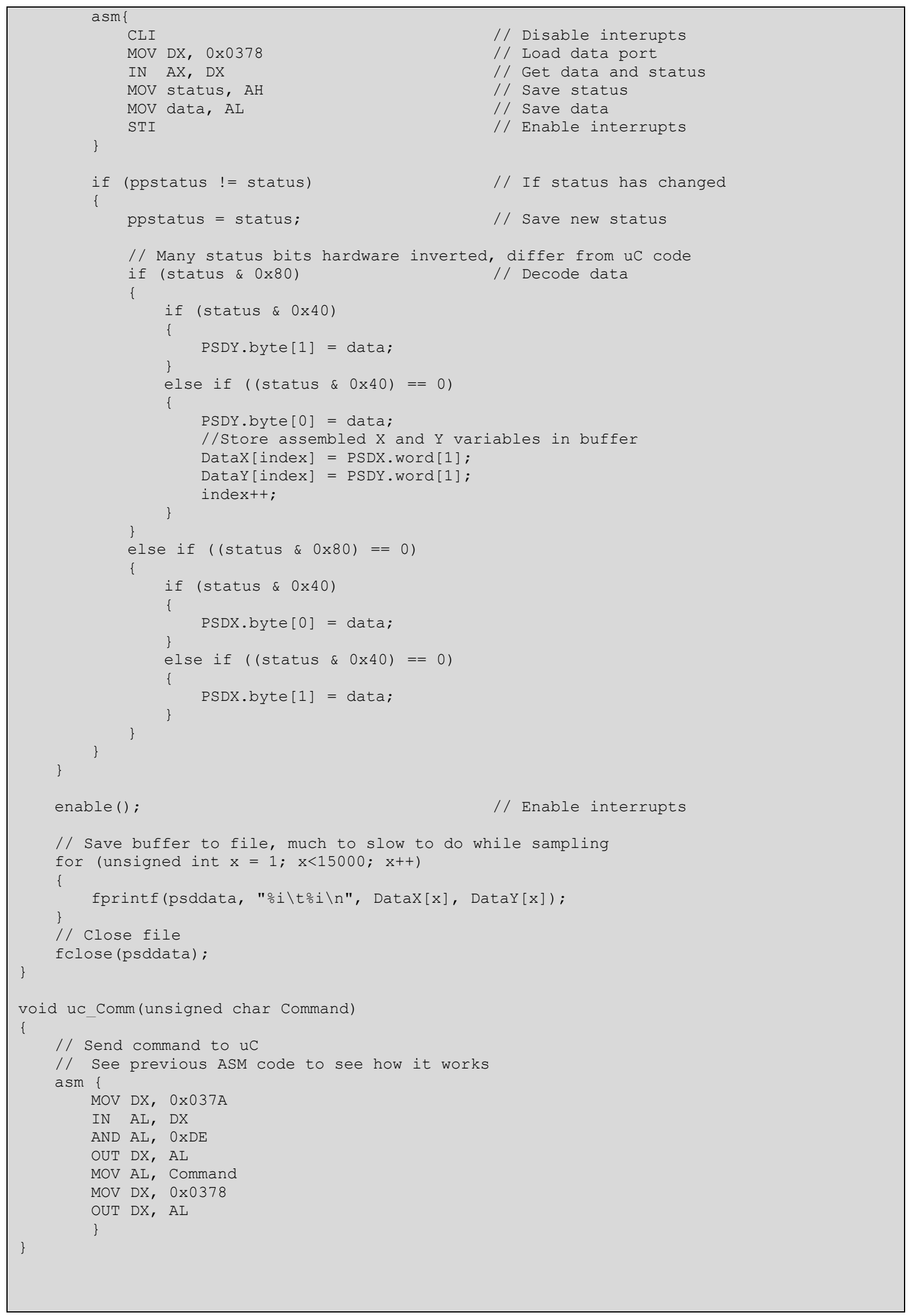


// A function prototype to enable the Serial Port

// This would be great to have working to enable to motor to run while the DAQ

// is sampling data. Should be relatively simple with a build in port command.

void Serial_Enable (void)

\{

asm

MOV DX, 0x03F9

MOV AL, $0 \times 00$

OUT DX, AL

MOV DX, 0x03FB

MOV AL, $0 \times 80$

// Set DLAB ON

OUT DX, AL

MOV DX, 0x03F8

MOV AL, OXOC

OUT DX, AL

MOV DX, Ox03F9

MOV AL, $0 \times 00$

OUT DX, AL

MOV DX, 0x03FB

MOV AL, $0 \times 03$

OUT DX, AL

MOV DX, 0x03FA

MOV AL, $0 \times C 7$

OUT DX, AL

MOV DX, Ox03FC

// Turn Off Interrupts

MOV AL, OXOB

OUT DX, AL

1

// Function to draw the nice graph on the screen

void Screen Setup(void)

\{

CLEAR SCREEN ();

int $x=0$;

for $(\mathrm{x}=10 ; \mathrm{x}<190 ; \mathrm{x}++)$

PIXEL $(160, x, 25)$;

for $(x=10 ; x<310 ; x++)$

PIXEL $(x, 100,25)$

for $(x=0 ; x<180 ; x++)$

$\operatorname{PIXEL}(70, x+10,20)$;

$\operatorname{PIXEL}(250, x+10,20)$;

\}

for $(x=0 ; x<310 ; x++)$

\{

$\operatorname{PIXEL}(x+10,10,20)$;

PIXEL $(x+10,190,20)$;

gotoxy $(3,25)$.

printf("PSD Data Acquisition Software") ; 


\section{APPENDIX B - Additional Figures}

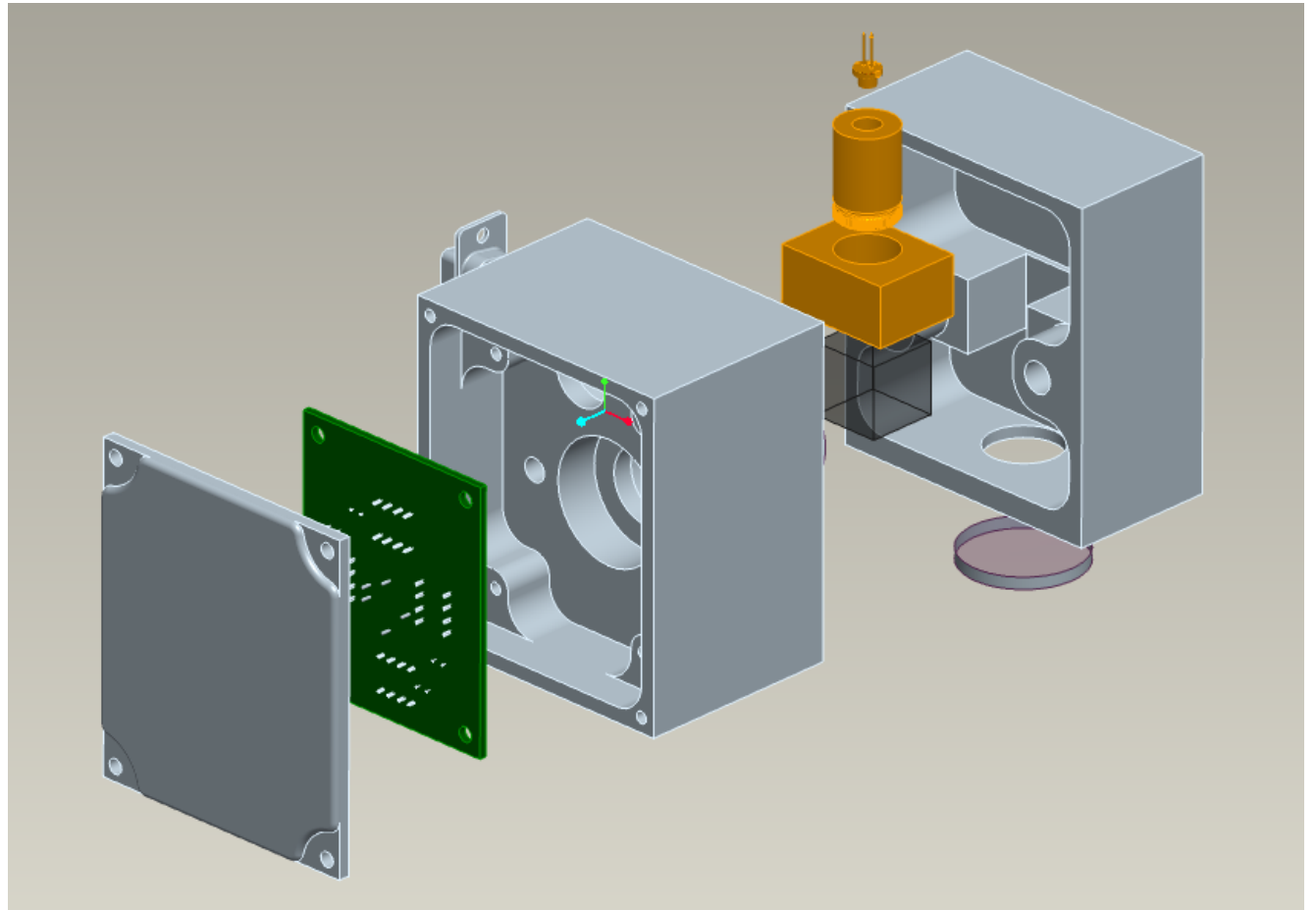

Figure B 1 - Exploded rear view of the optical housing. 


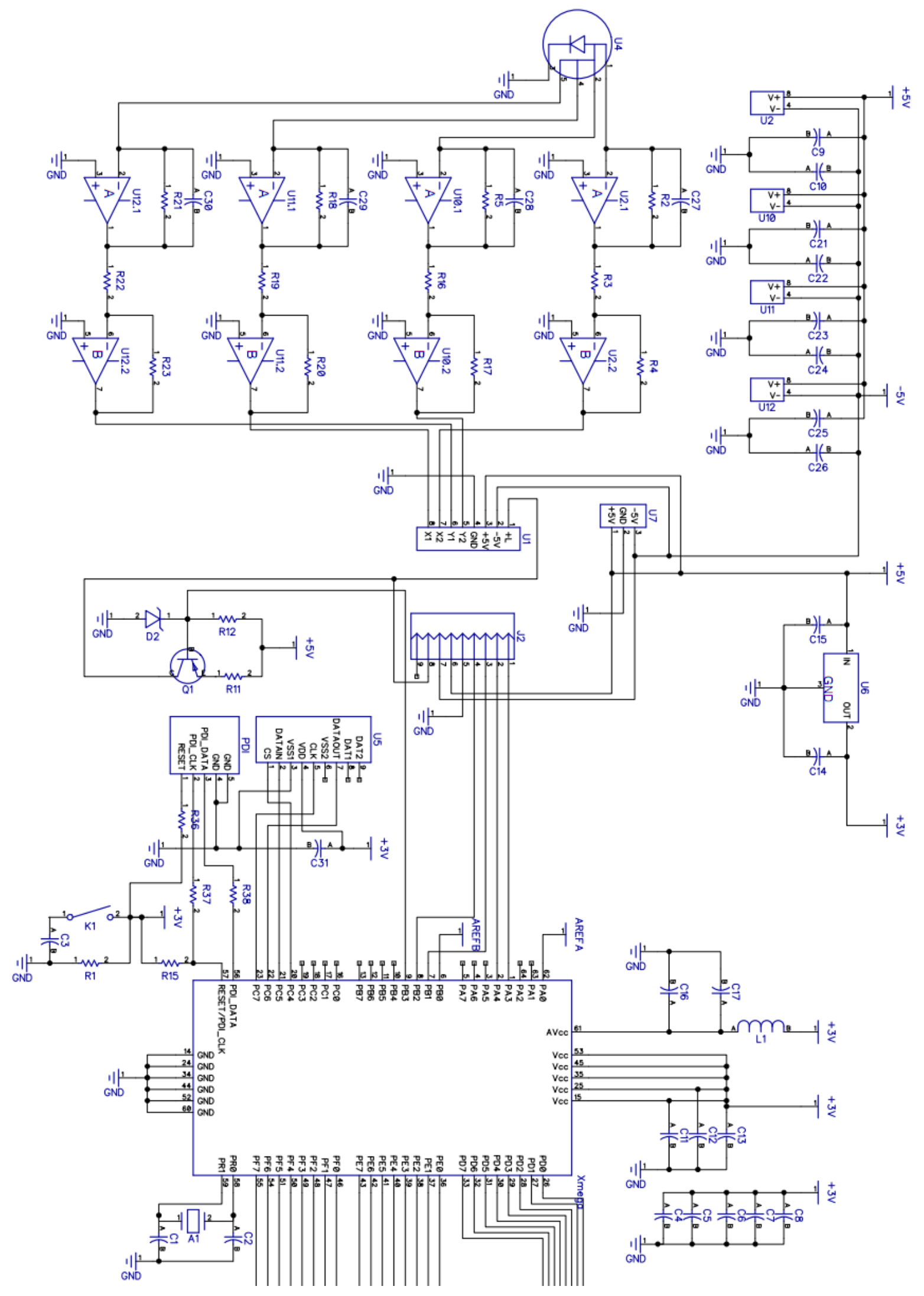




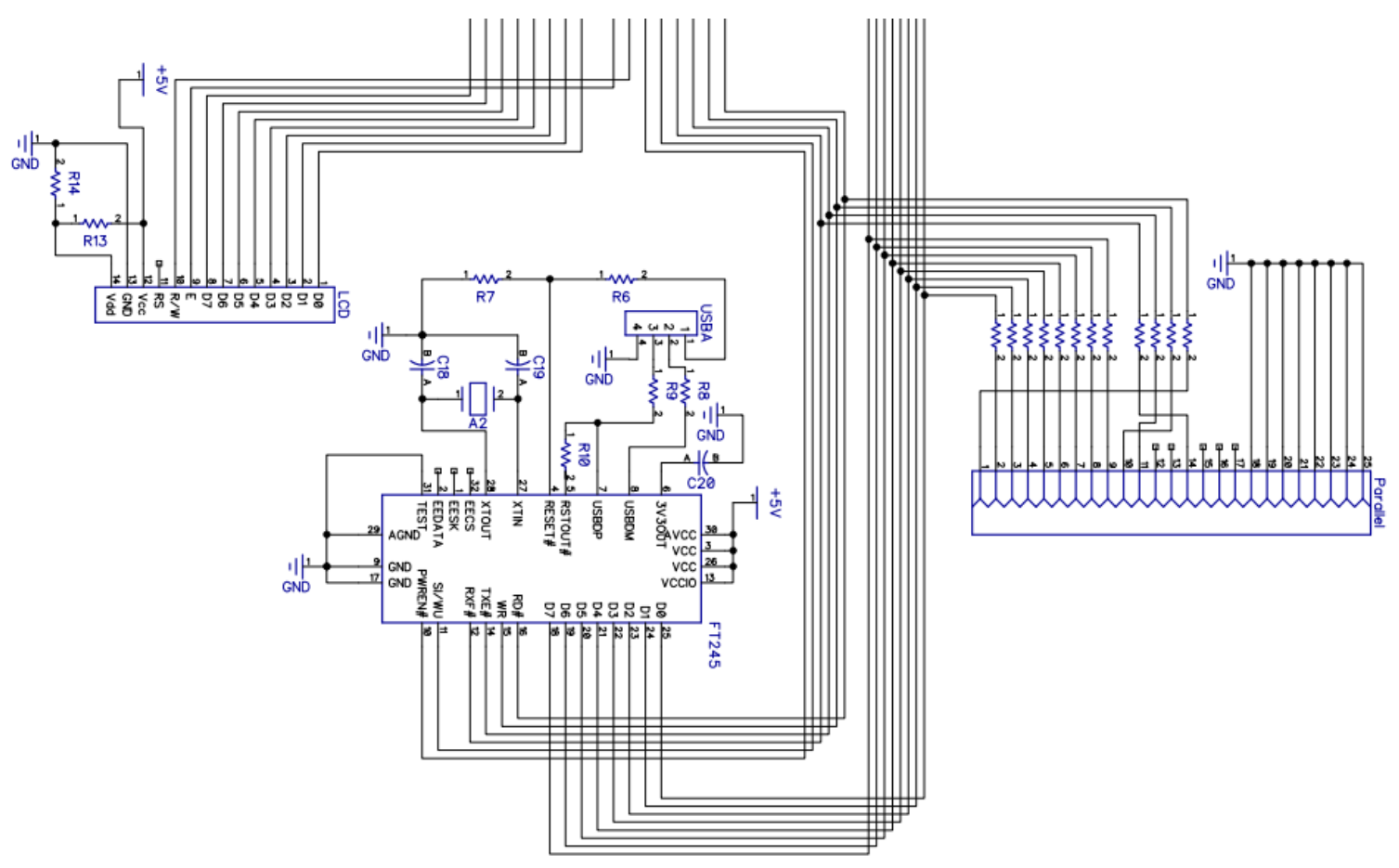

Figure B 2 - Electronics schematic of microcontroller and amplifier. 